\title{
Search for supersymmetry in events with at least three electrons or muons, jets, and missing transverse momentum in proton-proton collisions at $\sqrt{s}=13 \mathrm{TeV}$
}

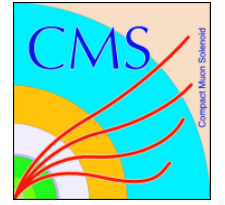

\section{The CMS collaboration}

E-mail: cms-publication-committee-chair@cern.ch

ABSTRACT: A search for new physics is carried out in events with at least three electrons or muons in any combination, jets, and missing transverse momentum. Results are based on the sample of proton-proton collision data produced by the LHC at a center-of-mass energy of $13 \mathrm{TeV}$ and collected by the CMS experiment in 2016. The data sample analyzed corresponds to an integrated luminosity of $35.9 \mathrm{fb}^{-1}$. Events are classified according to the number of $b$ jets, missing transverse momentum, hadronic transverse momentum, and the invariant mass of same-flavor dilepton pairs with opposite charge. No significant excess above the expected standard model background is observed. Exclusion limits at $95 \%$ confidence level are computed for four different supersymmetric simplified models with pair production of gluinos or third-generation squarks. In the model with gluino pair production, with subsequent decays into a top quark-antiquark pair and a neutralino, gluinos with masses smaller than $1610 \mathrm{GeV}$ are excluded for a massless lightest supersymmetric particle. In the case of bottom squark pair production, the bottom squark masses are excluded up to $840 \mathrm{GeV}$ for charginos lighter than $200 \mathrm{GeV}$. For a simplified model of heavy top squark pair production, the $\widetilde{t}_{2}$ mass is excluded up to 720,780 , or $710 \mathrm{GeV}$ for models with an exclusive $\widetilde{t}_{2} \rightarrow \widetilde{t}_{1} \mathrm{H}$ decay, an exclusive $\widetilde{\mathrm{t}}_{2} \rightarrow \widetilde{\mathrm{t}}_{1} \mathrm{Z}$ decay, or an equally probable mix of those two decays. In order to provide a simplified version of the analysis for easier interpretation, a small set of aggregate signal regions also has been defined, providing a compromise between simplicity and analysis sensitivity.

KEYWORDS: Hadron-Hadron scattering (experiments), Supersymmetry, Lepton production

ArXIV EPRINT: 1710.09154 


\section{Contents}

1 Introduction 1

2 The CMS detector 3

3 Event selection criteria and Monte Carlo simulation 3

$\begin{array}{lll}4 & \text { Search strategy } & 6\end{array}$

$\begin{array}{lll}5 & \text { Background estimation } & 7\end{array}$

$\begin{array}{llr}6 & \text { Systematic uncertainties } & 10\end{array}$

7 Results 13

8 Conclusions $\quad 20$

$\begin{array}{lr}\text { The CMS collaboration } & 27\end{array}$

\section{Introduction}

Many different theories beyond the standard model (BSM) predict processes leading to events containing multiple electrons and/or muons [1-5]. The background from standard model (SM) processes forging such a final state is small and dominated by multiboson production, which is well understood theoretically [6-20] and well reconstructed experimentally [21-25]. The search in this paper is designed to have broad sensitivity to a variety of BSM models by examining the event yields as a function of several kinematic quantities.

This paper describes the methods and results of a search for new physics in final states with three or more electrons or muons in any combination accompanied by jets and missing transverse momentum. A sample of proton-proton (pp) collision data, corresponding to an integrated luminosity of $35.9 \mathrm{fb}^{-1}$ and collected by the CMS detector at the CERN LHC at a center-of-mass energy of $13 \mathrm{TeV}$ throughout 2016, is used. Results of this analysis are interpreted in the context of supersymmetric (SUSY) models [26-34]. Supersymmetry is an extension of the SM that predicts a SUSY partner for every SM particle by introducing a new symmetry between bosons and fermions. It can potentially provide solutions to questions left open by the SM, such as the hierarchy problem and the nature of dark matter. More specifically, models in which $R$-parity [31] is conserved, whereby SUSY particles are produced only in pairs, can include a dark matter candidate in the form of a stable and undetectable lightest SUSY particle (LSP). In the models considered in this paper, the LSP is assumed to be the lightest neutralino (a mixture of the superpartners of the Higgs and $\mathrm{Z}$ bosons, and of the photon). 

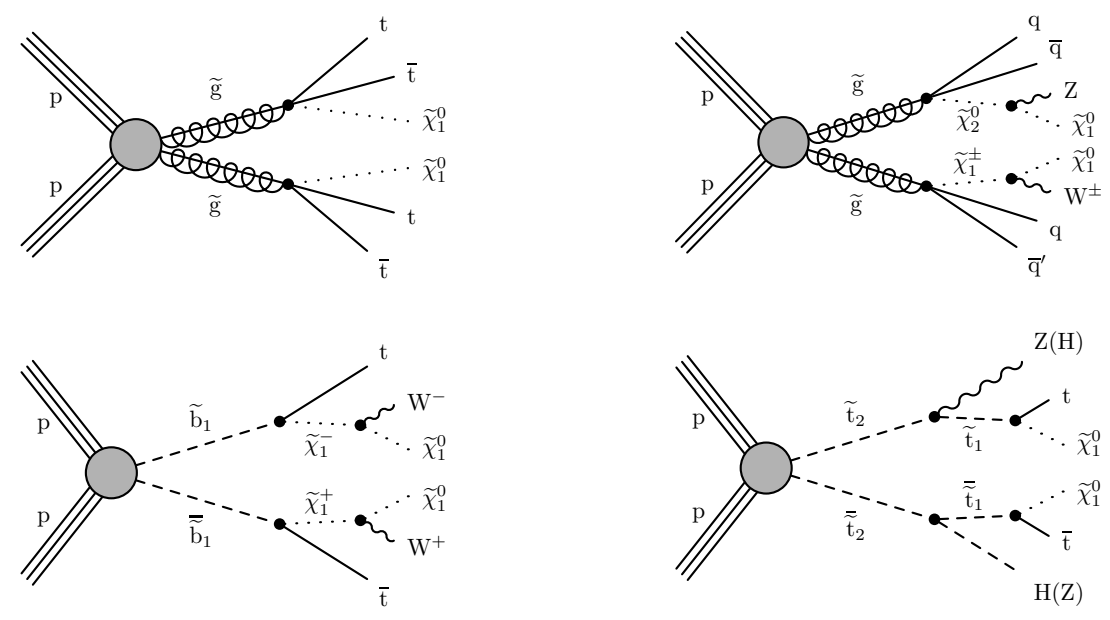

Figure 1. Diagrams for models with gluino pair production leading to four top quarks, T1tttt (upper left), or four quarks and two vector bosons, T5qqqqVV (upper right) in the final state, in both cases accompanied by two LSPs. Models of bottom, T6ttWW, and top squark, T6ttHZ, pair production lead to two top quarks, two LSPs and either two W bosons (lower left) or two neutral bosons as SM Higgs (H) and/or Z bosons (lower right).

The reference models for this analysis are simplified model spectra (SMS) [35]. Examples for SUSY processes that can give rise to multilepton final states are shown in figure 1. Throughout this paper lepton refers to an electron or a muon. The models under consideration in this analysis feature the pair production of gluinos, $\widetilde{g}$, or third generation squarks, $\widetilde{b}_{1}$ or $\widetilde{t}_{2}$, superpartners of gluons and third generation quarks, respectively, for a wide spectrum of possible masses. A typical process predicted by SUSY models consists of gluino pair production with each gluino decaying to a top quark pair, $t \bar{t}$, and an LSP, $\widetilde{\chi}_{1}^{0}$ (figure 1 , upper left), or to a pair of quarks and a neutralino, $\widetilde{\chi}_{2}^{0}$, or chargino, $\widetilde{\chi}_{1}^{ \pm}$. The latter would then decay into a $\mathrm{Z}$ or $\mathrm{W}$ boson, and an LSP (figure 1, upper right). The first model is referred to as T1tttt and the second one as T5qqqqVV throughout this paper. Other models feature bottom squark, $\widetilde{b}_{1}$, pair production, with subsequent cascade decays resulting in top quarks, W bosons and LSPs (figure 1, lower left) or pair production of the heaviest of the two top squark states, $\widetilde{\mathrm{t}}_{2}$, with subsequent decays to top quarks, Higgs or $\mathrm{Z}$ bosons, and LSPs (figure 1, lower right). The latter process allows a challenging scenario to be probed in which the mass difference between the lighter top squark, $\widetilde{\mathrm{t}}_{1}$, and the neutralino, $\widetilde{\chi}_{1}^{0}$, is close to the mass of the top quark [36, 37]. These two models are denoted as T6ttWW and T6ttHZ, respectively. Through the decays of $\mathrm{W}, \mathrm{Z}$ or Higgs bosons these processes can result in several leptons. In addition to the presence of multiple leptons, these models predict events with multiple jets and missing transverse momentum, largely induced by the undetected LSPs. The SUSY particles that are not directly included in the diagrams are assumed to be too heavy to be accessible at the LHC. Therefore, the only free parameters in these models are the mass of the produced gluinos or squarks, the masses of the possible intermediate particles in the decay chain, like $\widetilde{\chi}_{2}^{0}$ or $\widetilde{\chi}_{1}^{ \pm}$, and the mass of the $\widetilde{\chi}_{1}^{0}$. 
Similar searches have been carried out by the ATLAS and CMS Collaborations using the $13 \mathrm{TeV}$ dataset. With the data sample collected by the ATLAS experiment and corresponding to an integrated luminosity of $36.1 \mathrm{fb}^{-1}$, gluinos with masses up to $1870 \mathrm{GeV}$ can be excluded [38] assuming the model depicted in figure 1 (upper left). A comparable search at the same center-of-mass energy with the CMS detector in 2015, based on a data sample corresponding to an integrated luminosity of $2.3 \mathrm{fb}^{-1}$, excluded gluino masses below $1175 \mathrm{GeV}$ [39]. The current analysis improves upon the one performed with the data collected in 2015 with a more advanced strategy that exploits the transverse mass reconstructed with a lepton and the missing transverse momentum vector. Taking into account that approximately 15 times more data were collected in 2016, a new control region dominated by events from the t⿱亠幺ZZ process and a new interpretation of the results based on a T6ttHZ model also were added.

\section{The CMS detector}

The CMS detector features a superconducting solenoid with an internal diameter of $6 \mathrm{~m}$ that creates a magnetic field of $3.8 \mathrm{~T}$. Inside the magnet volume are a silicon pixel and strip tracker, an electromagnetic calorimeter (ECAL) made of lead tungstate crystals, and a hadronic calorimeter (HCAL) made of brass and scintillator material, each composed of a barrel and two endcap sections. Forward calorimeters extend the pseudorapidity $(\eta)$ coverage for the HCAL. In the barrel section of the ECAL, an energy resolution of about $1 \%$ is achieved for unconverted or late-converting photons in the tens of $\mathrm{GeV}$ energy range. The remaining barrel photons have a resolution of about $1.3 \%$ up to $|\eta|=1$, rising to about $2.5 \%$ at $|\eta|=1$.4. In the endcaps, the resolution of unconverted or late-converting photons is about $2.5 \%$, while the remaining endcap photons have a resolution between 3 and $4 \%$ [40]. When combining information from the entire detector, the jet energy resolution amounts typically to $15 \%$ at $10 \mathrm{GeV}, 8 \%$ at $100 \mathrm{GeV}$, and $4 \%$ at $1 \mathrm{TeV}$, to be compared to about $40 \%$, $12 \%$, and $5 \%$ obtained when the ECAL and HCAL calorimeters alone are used. Muons are measured in the range $|\eta|<2.4$, with detection planes made using three technologies: drift tubes, cathode strip chambers, and resistive plate chambers. Matching muons to tracks measured in the silicon tracker results in a relative transverse momentum resolution for muons with $20<p_{\mathrm{T}}<100 \mathrm{GeV}$ of $1.3-2.0 \%$ in the barrel and better than $6 \%$ in the endcaps, The $p_{\mathrm{T}}$ resolution in the barrel is better than $10 \%$ for muons with $p_{\mathrm{T}}$ up to $1 \mathrm{TeV}$ [41]. The first level of the CMS trigger system [42], composed of specialized hardware processors, uses information from the calorimeters and muon detectors to select the most interesting events in a fixed time interval of less than $4 \mu \mathrm{s}$. The high-level trigger (HLT) processor farm further decreases the event rate from approximately $100 \mathrm{kHz}$ to around $1 \mathrm{kHz}$, before the storage of the data. A more detailed description of the CMS detector, together with a definition of the coordinate system used and the relevant kinematic variables, can be found in $[43]$.

\section{Event selection criteria and Monte Carlo simulation}

Events are reconstructed using the particle flow, PF, algorithm [44], which reconstructs and identifies each individual particle with an optimized combination of information from the 
various elements of the CMS detector. The objects identified as particles by this algorithm are commonly referred to as PF candidates. Jets are clustered from PF candidates using the anti- $k_{\mathrm{T}}$ algorithm $[45,46]$ with a distance parameter of 0.4 . Only jets with transverse momentum $\left(p_{\mathrm{T}}\right)$ larger than $30 \mathrm{GeV}$ falling within $|\eta|<2.4$ are considered. To avoid double counting, the closest matching jets to leptons are not considered if they are separated from the lepton by less than 0.4 in $\Delta R \equiv \sqrt{(\Delta \eta)^{2}+(\Delta \phi)^{2}}$. Here $\Delta \eta$ and $\Delta \phi$ are the differences in $\eta$ and azimuthal angle ( $\phi$, in radians) between the considered lepton and a given jet. Additional criteria are applied to reject events containing noise and mismeasured jets. Jet energy scale (JES) corrections are applied to correct simulated jets for residual differences with data $[47,48]$.

The combined secondary vertex algorithm CSVv2 $[49,50]$ is used to assess the likelihood that a jet originates from a bottom quark. The tagging efficiency for true $b$ flavor jets is typically $70 \%$ and the misidentification probabilities are $10 \%$ and $1 \%$ for c quark and light-flavor jets, respectively. Jets with $p_{\mathrm{T}}>25 \mathrm{GeV}$ and within $|\eta|<2.4$ are considered for $\mathrm{b}$ tagging. Another variable related to jets that is used throughout this analysis is the scalar sum of the transverse momenta of all jets, defined as $H_{\mathrm{T}}=\sum_{\text {jets }} p_{\mathrm{T}}$, where jets have $p_{\mathrm{T}}>30 \mathrm{GeV}$. The missing transverse momentum $p_{\mathrm{T}}^{\text {miss }}$ is defined as the magnitude of $\vec{p}_{\mathrm{T}}^{\text {miss }}$, the negative vector sum of the transverse momenta all PF candidates reconstructed in an event $[51,52]$.

Electron candidates are reconstructed using tracking and ECAL information, by combining the clusters of energy deposits in the ECAL with Gaussian sum filter tracks [53]. The electron identification is performed using a multivariate discriminant built with shower shape variables, track-cluster matching variables, and track quality variables. The algorithm is optimized to select electrons from the decay of $\mathrm{W}$ and $\mathrm{Z}$ bosons with a $90 \%$ efficiency while rejecting electron candidates originating from jets. To reject electrons originating from photon conversions inside the detector, electrons are required to have all possible measurements in the innermost tracker layers and to be incompatible with any conversion-like secondary vertices. The identification of the muon is performed using the quality of the matching between the measurements of the tracker and the muon system [41]. The muon identification efficiency is at least $96 \%$, with some variation depending on $p_{\mathrm{T}}$ and $\eta$.

The reconstructed vertex with the largest value of summed physics object $p_{\mathrm{T}}^{2}$ is taken to be the primary pp interaction vertex. The physics objects are the objects returned by a jet finding algorithm [45, 46] applied to all charged tracks associated with the vertex, plus the corresponding associated missing transverse momentum. Both muon and electron candidates are required to have a transverse impact parameter smaller than $0.5 \mathrm{~mm}$ with respect to the primary vertex and a longitudinal impact parameter smaller than $1 \mathrm{~mm}$. In addition, a selection on the three-dimensional impact parameter significance, defined as the value of impact parameter divided by its uncertainty, is applied. This value has to be smaller than 4 for both electrons and muons.

Additional information about the isolation of the lepton is necessary to discriminate between leptons originating from decays of heavy particles such as W and Z bosons ("prompt" leptons) and those produced in hadron decays or jets misidentified as leptons ("nonprompt" leptons). The lepton isolation criterion is constructed using three different variables. 
The relative isolation, $I_{\mathrm{rel}}$, is defined as the ratio of the amount of energy measured in a cone around the lepton to the $p_{\mathrm{T}}$ of the lepton, $p_{\mathrm{T}}^{\ell}$, with a $p_{\mathrm{T}}^{\ell}$-dependent radius [54]:

$$
\Delta R \leq \frac{10 \mathrm{GeV}}{\min \left(\max \left(p_{\mathrm{T}}^{\ell}, 50 \mathrm{GeV}\right), 200 \mathrm{GeV}\right)}
$$

Requiring $I_{\text {rel }}$ below a given threshold ensures that the lepton is locally isolated, even in Lorentz-boosted topologies.

The second isolation variable is the ratio of the lepton $p_{\mathrm{T}}$ and that of the jet geometrically closest to the lepton: $p_{\mathrm{T}}^{\text {ratio }}=p_{\mathrm{T}}^{\ell} / p_{\mathrm{T}}^{\text {jet }}$. In most cases this is the jet containing the lepton. If no jet is found within a cone defined by $\Delta R<0.4$, the ratio is set to 1 . The use of $p_{\mathrm{T}}^{\text {ratio }}$ provides a way to identify nonprompt low- $p_{\mathrm{T}}$ leptons originating from low- $p_{\mathrm{T}}$ b jets, which decay with a larger opening angle than the one used in $I_{\text {rel }}$.

The last variable used in the isolation criteria of leptons is $p_{\mathrm{T}}^{\mathrm{rel}}$, defined as the magnitude of the component of the lepton momentum perpendicular to the axis of the closest jet. The jet axis is obtained by subtracting the momentum vector of the lepton from that of the jet. If no matched jet is found around the lepton, the variable is set to 0 . This variable allows the recovery of leptons from accidental overlap with jets in Lorentz-boosted topologies. For the calculation of $p_{\mathrm{T}}^{\text {rel }}$ and the previously mentioned $p_{\mathrm{T}}^{\text {ratio }}$, jets with $p_{\mathrm{T}}>5 \mathrm{GeV}$ and without any additional identification criteria are considered.

Using those three variables, a lepton is considered isolated if the following condition is fulfilled:

$$
I_{\text {rel }}<I_{1} \operatorname{AND}\left(p_{\mathrm{T}}^{\text {ratio }}>I_{2} \text { OR } p_{\mathrm{T}}^{\text {rel }}>I_{3}\right) .
$$

The values of $I_{1}, I_{2}$, and $I_{3}$ depend on the flavor of the lepton; the probability to misidentify a jet as a lepton is higher for electrons than for muons, so tighter isolation values are used for the former. For electrons (muons), the tight selection requirements are $I_{1}=0.12(0.16), I_{2}=0.76(0.69)$, and $I_{3}=7.2(6.0) \mathrm{GeV}$. The isolation requirement for leptons to pass the loose working point of the selection is significantly relaxed, only consisting of $I_{\text {rel }}<0.4$.

Events used in this analysis are required to pass trigger selection criteria that target dilepton and multilepton events. The following two sets of triggers are used in a logic OR configuration. One set of triggers requires that the two leptons satisfy loose isolation criteria and that the highest- $p_{\mathrm{T}}$ (leading) lepton have $p_{\mathrm{T}}>23(17) \mathrm{GeV}$ and the second highest- $p_{\mathrm{T}}$ (sub-leading) lepton have $p_{\mathrm{T}}>12(8) \mathrm{GeV}$ for muons (electrons). The second set of triggers places no requirements on the isolation, has a lower $p_{\mathrm{T}}$ threshold for both leptons $\left(p_{\mathrm{T}}>8 \mathrm{GeV}\right)$, and requires the $H_{\mathrm{T}}$ reconstructed in the trigger to be greater than $300 \mathrm{GeV}$. With the thresholds on the $p_{\mathrm{T}}$ of the leptons and on the $H_{\mathrm{T}}$ applied, the efficiency per event is near $100 \%$.

The selection requires the presence of at least three well-identified leptons in the event. The leptons must satisfy $p_{\mathrm{T}}$ thresholds that depend on the lepton flavor and the amount of hadronic activity in the event. For events with low hadronic activity $\left(H_{\mathrm{T}}<400 \mathrm{GeV}\right)$, the leading electron (muon) must satisfy $p_{\mathrm{T}}>25(20) \mathrm{GeV}$ and sub-leading electrons (muons) must satisfy $p_{\mathrm{T}}>15(10) \mathrm{GeV}$. In events with high hadronic activity $\left(H_{\mathrm{T}}>400 \mathrm{GeV}\right)$, 
the thresholds are relaxed to $15(10) \mathrm{GeV}$ for the leading electrons (muons). The lowest$p_{\mathrm{T}}$ (trailing) lepton must have $p_{\mathrm{T}}>10 \mathrm{GeV}$ in all cases. Opposite-charge same-flavor lepton pairs are required to have an invariant mass $\left(m_{\ell \ell}\right)$ greater than $12 \mathrm{GeV}$ to suppress Drell-Yan and quarkonium processes.

In order to estimate the contribution from SM processes with prompt leptons in the signal regions and to calculate the predicted yields from new physics models, Monte Carlo (MC) simulations are used. The MADGRAPH5_amC@NLOv2.2.2 or v2.3.3 generator [55] was used to simulate events for the $\mathrm{t} \overline{\mathrm{t}}, \mathrm{W} \gamma^{*}$ and $\mathrm{tWZ}$ processes, at leading order (LO), and for $\mathrm{t} \overline{\mathrm{tZ}}, \mathrm{t \overline { \textrm {t } }}$, $\mathrm{tZq}, \mathrm{tHq}, \mathrm{tHW}, \mathrm{WWZ}, \mathrm{WZZ}, \mathrm{ZZZ}, \mathrm{t} \overline{\mathrm{t}} \gamma$, and $\mathrm{Z} \gamma^{*}$ final states, at next-to-leading order (NLO) in perturbative quantum chromodynamics. The NLO POWHEG v2 [56] gener-

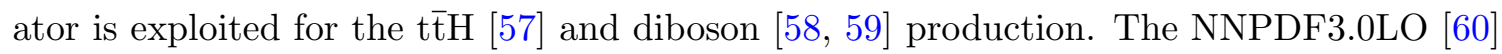
parton distribution functions (PDFs) are used for the simulated samples generated at LO and the NNPDF3.0NLO [60] PDFs for those generated at NLO. Parton showering and hadronization are simulated using the PYTHIA v8.212 generator [61] with the CUETP8M1 tune $[62,63]$. A double-counting of the partons generated with MADGRAPH5_aMC@NLOand those with PYTHIA is removed using the MLM [64] and the FXFX [65] matching schemes, in the LO and NLO samples, respectively. The CMS detector response is modeled using a GEANT4-based model [66]. The simulated samples include additional simultaneous interactions per bunch crossing (pileup), with distributions that are weighted to match the observed data.

Monte Carlo simulation of signal events used for interpretation of the final results is done with the MADGRAPH5_aMC@NLO program at LO precision, allowing for up to two additional partons in the calculation of the matrix elements. The SUSY particle decays, parton showering, and hadronization are simulated with PYTHIA v8.212. The detector response for signal events is simulated using a CMS fast-simulation package [67] that is validated with respect to the GEANT4-based model. All simulated events are processed with the same reconstruction procedure as data. Cross sections for SUSY signal processes, calculated at NLO with next-to-leading-logarithmic (NLL) resummation, were provided by the LHC SUSY Cross section Working Group [68-73].

\section{Search strategy}

A baseline selection is applied to the dataset containing events of interest: three or more electrons or muons, at least two jets $\left(N_{\text {jets }} \geq 2\right), p_{\mathrm{T}}^{\text {miss }} \geq 50 \mathrm{GeV}$, and $m_{\ell \ell} \geq 12 \mathrm{GeV}$ for all opposite-charge, same-flavor lepton pairs. All these requirements are listed in table 1. Two different regions are defined, based on whether or not an event contains an oppositecharge, same-flavor lepton pair with an invariant mass within the $15 \mathrm{GeV}$ window around the $\mathrm{Z}$ boson mass [74]. If such a lepton pair is found the event is categorized as "on-Z", otherwise "off-Z".

Events are further categorized into signal regions, which are defined according to several event observables: $N_{\mathrm{b}}$ jets $, H_{\mathrm{T}}, p_{\mathrm{T}}^{\text {miss }}, \mathrm{m}_{\ell \ell}$, as well as the transverse mass reconstructed with a lepton and the missing transverse momentum vector,

$$
M_{\mathrm{T}}=\sqrt{2 p_{\mathrm{T}}^{\ell} p_{\mathrm{T}}^{\mathrm{miss}}\left[1-\cos \left(\phi_{\ell}-\phi_{\vec{p}_{\mathrm{T}}^{\mathrm{miss}}}\right)\right]} .
$$




\begin{tabular}{|lc|}
\hline Number of selected leptons & $\geq 3$ \\
$N_{\text {jets }}$ & $\geq 2$ \\
$p_{\mathrm{T}}^{\text {miss }}, \mathrm{GeV}$ & $>50\left(70\right.$ in low $N_{\mathrm{b} \text { jets }}$ and low $H_{\mathrm{T}}$ category $)$ \\
$\mathrm{m}_{\ell \ell}, \mathrm{GeV}$ & $>12$ \\
\hline
\end{tabular}

Table 1. Summary of all requirements used in baseline selection criteria.

If the event is categorized as on-Z, the $M_{\mathrm{T}}$ is calculated with the lepton that is not involved in the $\mathrm{Z}$ boson mass reconstruction, otherwise the lepton yielding the lowest $M_{\mathrm{T}}$ value $\left(M_{\mathrm{T}}^{\min }\right)$ is used in the computation of this variable.

The classification of selected events based on the number of $b$ jets creates signal regions with high signal-to-background ratios for events from different signal models. For example, the T1tttt model features several $b$ jets, which would be categorized into signal regions that are almost free of the leptonic WZ background owing to the $\mathrm{b}$ jet requirements. Including the $0 \mathrm{~b}$ jet signal regions keeps the analysis sensitive to signatures without $\mathrm{b}$ jets, such as T5qqqqVV model. Additionally, a categorization in $H_{\mathrm{T}}$ and $p_{\mathrm{T}}^{\text {miss }}$ is useful to distinguish between compressed and noncompressed SUSY spectra, i.e. models with small or large mass differences between the SUSY particles in the decay chain.

Table 2 shows the definition of the signal regions (SRs) into which the events passing the baseline selection are subdivided. There are 16 separate off-Z and 16 on-Z SRs. Each category is split, depending on the number of b jets $\left(0,1\right.$ and 2), the value of $H_{\mathrm{T}}$ (greater or lower than $400 \mathrm{GeV}$ ), and $p_{\mathrm{T}}^{\text {miss }}$ (greater or lower than $150 \mathrm{GeV}$ ). These SRs are denoted as SR 1-12. Motivated by the low expected yield of events with high $\mathrm{b}$ jet multiplicities, one inclusive SR with $p_{\mathrm{T}}^{\text {miss }}<300 \mathrm{GeV}$ and $H_{\mathrm{T}}<600 \mathrm{GeV}$ has been defined for $\geq 3$ b jets (SR 13), and additionally to this three SRs with significant amounts of $H_{\mathrm{T}}$ (>600 GeV, SRs 14 , 15) or $p_{\mathrm{T}}^{\text {miss }}(>300 \mathrm{GeV}$, SR 16) have been introduced, since various noncompressed SUSY models yield very high values for these variables. These latter three regions are inclusive in the number of $\mathrm{b}$ jets. All of the $0 \mathrm{~b}$ jet regions, as well as three regions with high $H_{\mathrm{T}}$ and $p_{\mathrm{T}}^{\text {miss }}$ values, are further split depending whether $M_{\mathrm{T}}$ is smaller (designated with the letter "a" after the region number) or greater (designated with "b") than $120 \mathrm{GeV}$, leading to a total of 23 regions for each of the off- $Z$ and on- $Z$ categories. In the on- $Z$ regions with 0 or $1 \mathrm{~b}$ jet and $60<H_{\mathrm{T}}<400 \mathrm{GeV}$, the $p_{\mathrm{T}}^{\text {miss }}$ lower bound is raised to $70 \mathrm{GeV}$ to completely suppress the contribution from the Drell-Yan process.

In order to provide a simplified version of the analysis for easier interpretation, a small set of aggregate signal regions has been defined, providing a compromise between simplicity and analysis sensitivity. The definition of these so-called super signal regions (SSR) is given in table 3 . The additional requirement $M_{\mathrm{T}}$ greater than $120 \mathrm{GeV}$ was added to the SSRs with respect to the relevant SRs.

\section{Background estimation}

All backgrounds leading to the multilepton final states targeted by this analysis can be subdivided into the categories listed below. 


\begin{tabular}{|c|c|c|c|c|c|}
\hline$N_{\text {jets }}$ & $N_{\mathrm{b} \text { jets }}$ & $H_{\mathrm{T}}[\mathrm{GeV}]$ & $50(70) \leq p_{\mathrm{T}}^{\text {miss }}<150 \mathrm{GeV}$ & $150 \leq p_{\mathrm{T}}^{\text {miss }}<300 \mathrm{GeV}$ & $p_{\mathrm{T}}^{\mathrm{miss}} \geq 300 \mathrm{GeV}$ \\
\hline \multirow{8}{*}{$\geq 2$} & & $60-400$ & SR1 † & $\mathrm{SR} 2 \dagger$ & \multirow{8}{*}{$\mathrm{SR} 16 \dagger$} \\
\hline & 0 & $400-600$ & $\mathrm{SR} 3 \dagger$ & $\mathrm{SR} 4 \dagger$ & \\
\hline & 1 & $60-400$ & SR5 & SR6 & \\
\hline & 1 & $400-600$ & SR7 & SR8 & \\
\hline & ? & $60-400$ & SR9 & SR10 & \\
\hline & 2 & $400-600$ & SR11 & SR12 & \\
\hline & $\geq 3$ & $60-600$ & \multicolumn{2}{|c|}{ SR13 } & \\
\hline & inclusive & $\geq 600$ & SR14 † & SR15 † & \\
\hline
\end{tabular}

Table 2. Summary of the signal region definitions. The minimum $p_{\mathrm{T}}^{\mathrm{miss}}$ requirement is raised from 50 to $70 \mathrm{GeV}$ only for the on-Z SR1 and SR5. Signal regions that are further subdivided at $M_{\mathrm{T}}=$ $120 \mathrm{GeV}$ are indicated with $\dagger$. The search regions are mirrored for on- and off-Z categories.

\begin{tabular}{|c|c|c|}
\hline \multirow{4}{*}{ off-Z } & $N_{\mathrm{b} \text { jets }} \leq 2, \quad M_{\mathrm{T}}^{\text {min }} \geq 120 \mathrm{GeV}$ & $N_{\mathrm{b} \text { jets }} \geq 3, \quad M_{\mathrm{T}}^{\text {min }} \geq 120 \mathrm{GeV}$ \\
& $H_{\mathrm{T}} \geq 200 \mathrm{GeV}, \quad p_{\mathrm{T}}^{\text {miss }} \geq 250 \mathrm{GeV}$ & $H_{\mathrm{T}} \geq 60 \mathrm{GeV}, \quad p_{\mathrm{T}}^{\text {miss }} \geq 50 \mathrm{GeV}$ \\
\cline { 2 - 3 } on-Z & SSR1 & SSR2 \\
\hline
\end{tabular}

Table 3. Definition of the aggregate super signal regions (SSRs). This simpler classification is proposed for reinterpretations, depending on the presence of a $\mathrm{Z}$ boson candidate and the number of $\mathrm{b}$ jets, along with additional simultaneous requirements on $M_{\mathrm{T}}, p_{\mathrm{T}}^{\mathrm{miss}}$, and $H_{\mathrm{T}}$.

Nonprompt leptons are leptons from heavy-flavor decays, misidentified hadrons, muons from light-meson decays in flight, or electrons from unidentified photon conversions. In this analysis $t \bar{t}$ events can enter the signal regions if nonprompt leptons are present in addition to the prompt leptons from the $\mathrm{W}$ boson decays. Top quark pair production gives the largest contribution for regions with low $H_{\mathrm{T}}$ and $p_{\mathrm{T}}^{\text {miss }}$ values, and therefore predominately

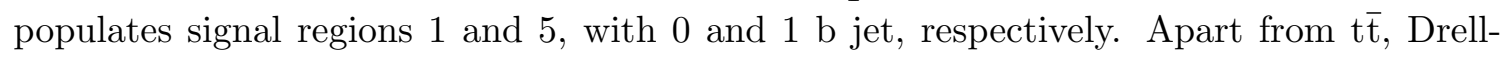
Yan events can enter the baseline selection. However, they are largely suppressed by the $p_{\mathrm{T}}^{\text {miss }}>50 \mathrm{GeV}$ selection, and additional rejection is achieved by increasing the $p_{\mathrm{T}}^{\text {miss }}$ requirement to $70 \mathrm{GeV}$ for on- $\mathrm{Z}$ regions with low $H_{\mathrm{T}}$ and low $p_{\mathrm{T}}^{\text {miss }}$. Processes that yield only one prompt lepton in addition to nonprompt ones, such as $\mathrm{W}+$ jets and various single top quark channels, are effectively suppressed by the three-lepton requirement because of the low probability that two nonprompt leptons satisfy the tight identification and isolation requirements. Albeit small, this contribution is nevertheless accounted for in our method to estimate the background due to nonprompt leptons (see below).

Diboson production can yield multilepton final states with up to three prompt leptons (WZ or $\left.\mathrm{W} \gamma^{*}\right)$ and up to four prompt leptons $\left(\mathrm{ZZ}\right.$ or $\left.\mathrm{Z} \gamma^{*}\right)$, rendering irreducible backgrounds for this analysis. For simplicity, in the following we refer to these backgrounds as WZ and ZZ, respectively. The WZ production has a sizable contribution in the on- $\mathrm{Z}$ events, especially in the SRs without b jets. The yields of these backgrounds in the various SRs are estimated by means of MC simulation, with the normalization factors derived from control regions in data. 


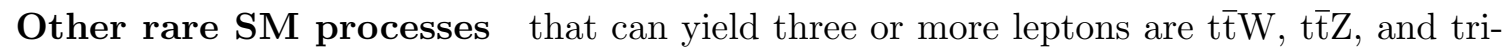
boson production. We also include the contribution from the SM Higgs boson produced in association with a vector boson or a pair of top quarks in this category of backgrounds, as well as processes that produce additional leptons from internal conversions, which are events that contain a virtual photon that decays to leptons. The internal conversion background components, $\mathrm{X}+\gamma$, are strongly suppressed by the $p_{\mathrm{T}}^{\text {miss }}>50 \mathrm{GeV}$ and $N_{\text {jets }} \geq 2$ requirements. The background events containing top quark(s) in association with a $\mathrm{W}, \mathrm{Z}$

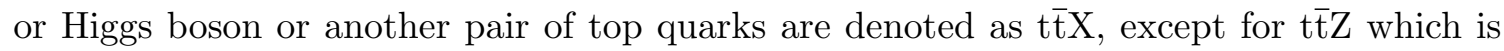
separately delineated. For the estimation of the latter process, the same strategy as for the WZ is used. All other processes are grouped into one category that is denoted as rare $\mathrm{SM}$ processes. The contribution from these processes as well as $\mathrm{t} \overline{\mathrm{t}} \mathrm{X}$ are estimated from MC simulation.

The background contribution from nonprompt leptons is estimated using the tight-toloose ratio method [54]. In this method, the yield is estimated in an application region that is similar to the signal region but which contains at least one lepton that fails the tight identification and isolation requirements but satisfies the loose requirements. The events in this region are weighted by $f /(1-f)$, where the tight-to-loose ratio $f$ is the probability that a loosely identified lepton also satisfies the full set of requirements. This ratio is measured as a function of lepton $p_{\mathrm{T}}$ and $\eta$ in a control sample of multijet events that is enriched in nonprompt leptons (measurement region). In this region, we require exactly one lepton, satisfying the loose object selection, and one recoiling jet with $\Delta R($ jet,$\ell)>1.0$ and $p_{\mathrm{T}}>30 \mathrm{GeV}$ in the event. To suppress processes that can contribute prompt leptons from a $\mathrm{W}$ or $\mathrm{Z}$ boson decay, such as $\mathrm{W}(+$ jets $)$, $\mathrm{DY}$ or $\mathrm{t} \overline{\mathrm{t}}$, we additionally require both $p_{\mathrm{T}}^{\text {miss }}$ and $M_{\mathrm{T}}$ to be below $20 \mathrm{GeV}$. The remaining contribution from these processes within the measurement region is estimated from MC simulation and subsequently subtracted from the data.

In order to reduce the dependence of the tight-to-loose ratio on the flavor composition of the jets from which the nonprompt leptons originate, this ratio is parameterized as a function of a variable that correlates more strongly with the mother parton $p_{\mathrm{T}}$ than with the lepton $p_{\mathrm{T}}$. This variable is calculated by correcting the lepton $p_{\mathrm{T}}$ as a function of the energy in the isolation cone around it. This definition leaves the $p_{\mathrm{T}}$ of the leptons satisfying the tight isolation criteria unchanged and modifies the $p_{\mathrm{T}}$ of those failing these criteria so that it is a better proxy for the mother parton $p_{\mathrm{T}}$ and results in a smaller variation as a function of the mother parton $p_{\mathrm{T}}$. The flavor dependence, which is much more important for the case of electrons, is further reduced by adjusting the loose electron selection to obtain similar $f$ values for nonprompt electrons that originate from light- or heavy-flavor jets. As a result, the tight-to-loose ratio measured in a multijet sample leads to a good description of nonprompt background originating from $t \bar{t}$ events, which in most of the SR are dominant in this category of background.

The tight-to-loose ratio method for estimating the nonprompt background is validated both in a closure test in simulation and in a data control region orthogonal to the baseline selection with minimal signal contamination. This region is defined by the requirement of three leptons that satisfy the nominal identification, isolation, and $p_{\mathrm{T}}$ selection, one or two 
jets, $30<p_{\mathrm{T}}^{\text {miss }}<50 \mathrm{GeV}$, and no dilepton pair with an invariant mass compatible with a $\mathrm{Z}$ boson. With these selection criteria a purity in $\mathrm{t} \overline{\mathrm{t}}$ of $80 \%$ can be achieved. We find an agreement of the order of $20-30 \%$ between the predicted and observed yields in this control region.

The WZ process is one of the main backgrounds in the regions with $0 \mathrm{~b}$ jets, while t $\overline{\mathrm{t} Z}$ gives a significant contribution in categories enriched in $\mathrm{b}$ jets. As mentioned earlier, the contribution of these backgrounds is estimated from simulation, but their normalizations are obtained from a simultaneous fit using two control regions, designed so that each is highly enriched in one of the processes. The WZ control region is defined by the requirement of three leptons satisfying the nominal identification and isolation selections. Two leptons have to form an opposite charge, same flavor pair with $\left|m_{\ell \ell}-m_{\mathrm{Z}}\right|<15 \mathrm{GeV}$, the number of jets and $\mathrm{b}$ jets has to be $\leq 1$ and 0 , respectively. The $p_{\mathrm{T}}^{\text {miss }}$ has to be in the range $30<$ $p_{\mathrm{T}}^{\text {miss }}<100 \mathrm{GeV}$, and $M_{\mathrm{T}}$ is required to be at least $50 \mathrm{GeV}$ to suppress contamination from the Drell-Yan process. The purity of the WZ control region is $80 \%$. The orthogonal control region for $\mathrm{t} \overline{\mathrm{tZ}}$ is defined similarly to that for $\mathrm{WZ}$, except for a requirement on the number of jets: three leptons satisfying the nominal identification and isolation selection are to be found, two of them forming an opposite charge, same flavor pair with $\left|m_{\ell \ell}-m_{\mathrm{Z}}\right|<15 \mathrm{GeV}$, at least 3 jets, and $30<p_{\mathrm{T}}^{\text {miss }}<50 \mathrm{GeV}$. Events are classified by the number of $\mathrm{b}$ jets, and three bins are formed for the tẼZ CR: the $0 \mathrm{~b}$ jet category, where the background is dominated by the WZ and t $\overline{\mathrm{t}}$ processes, and the 1 and $\geq 2 \mathrm{~b}$ jet categories, enriched in $\mathrm{t} \overline{\mathrm{t} Z}$.

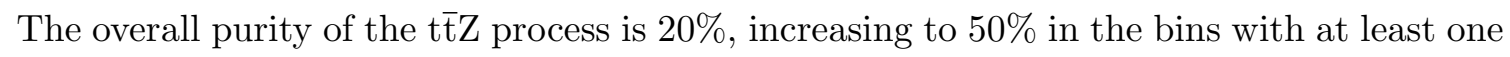
$\mathrm{b}$ jet. These three bins, together with the WZ control region are used in a simultaneous fit to obtain the scale factors for the normalization of the simulated samples. In the fit to data, the normalization and relative population across all four bins of all the components are allowed to vary according to experimental and theoretical uncertainties. For the WZ process the obtained scale factor is compatible with unity, $1.01 \pm 0.07$, and no correction is applied to the simulation, while for the t昏 it is found to be $1.14 \pm 0.28$. Therefore the yields from the $\mathrm{MC} \mathrm{t} \overline{\mathrm{t}} \mathrm{Z}$ sample obtained in the baseline region are scaled by a factor of 1.14 .

\section{Systematic uncertainties}

The uncertainties in the expected SM backgrounds and signal yields are categorized as experimental, such as those related to the JES or the b tagging efficiency description in the simulation; theoretical, such as the uncertainties in the considered cross sections; statistical, related to the observed yield in control regions in data; and as uncertainties in the background estimation methods relying on control regions in data. These uncertainties and their effect on the predicted yields are described below and summarized in table 4 .

One of the major experimental sources of uncertainty is the knowledge of the JES. This uncertainty affects all simulated background and signal events. For the data set used in this analysis, the uncertainties in the jet energy scale vary from $1 \%$ to $8 \%$, depending on the transverse momentum and pseudorapidity of the jet. The impact of these uncertainties is assessed by shifting the jet energy correction factors for each jet up and down by one standard deviation and recalculating all kinematic quantities. The systematic uncertainties 


\begin{tabular}{|c|c|c|}
\hline Source & Effect on the backgrounds [\%] & Effect on signal [\%] \\
\hline Integrated luminosity & 2.5 & 2.5 \\
\hline JES & $1-8$ & $1-10$ \\
\hline b tag efficiency & $1-8$ & $1-10$ \\
\hline Pileup & $1-5$ & $1-5$ \\
\hline Lepton efficiencies & 9 & 15 \\
\hline HLT efficiencies & 3 & 3 \\
\hline Nonprompt application region statistics & $10-100$ & - \\
\hline Nonprompt extrapolation & 30 & - \\
\hline WZ control region normalization & 10 & - \\
\hline t€̇Z control region normalization & 25 & - \\
\hline Limited size of simulated samples & $1-100$ & $10-100$ \\
\hline ISR modeling & - & $1-10$ \\
\hline Modeling of unclustered energy & - & $1-20$ \\
\hline 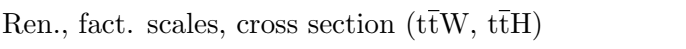 & $11-13$ & - \\
\hline 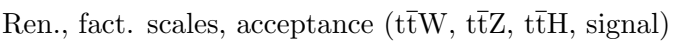 & $3-18$ & $3-18$ \\
\hline 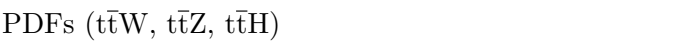 & $2-3$ & - \\
\hline Other rare backgrounds & 50 & - \\
\hline
\end{tabular}

Table 4. The effect of the systematic uncertainties on the event yields of the backgrounds and signal processes.

related to JES corrections are also propagated to the $p_{\mathrm{T}}^{\text {miss }}$ calculation. The propagation of the variation of the JES results in a variation of $1-10 \%$ in the predicted event yields in the various signal regions of this analysis.

A similar approach is used for the uncertainties associated with the corrections for the $\mathrm{b}$ tagging efficiencies for light, charm and bottom flavor jets, which are parameterized as a function of $p_{\mathrm{T}}$ and $\eta$. The variation of the scale factor correcting for the differences between data and simulation is at a maximum of the order of $10 \%$ per jet, and leads to an overall effect in the range of $1-10 \%$ depending on the signal region and on the topology of the event. The inaccuracy of the inelastic cross section value that affects the pile up rate gives up to a $5 \%$ effect. The sources of uncertainties explained here were also studied for the signal samples, and their impact on the predicted signal yields in every search region has been estimated following the same procedures.

Lepton identification and isolation scale factors have been measured as a function of lepton $p_{\mathrm{T}}$ and $\eta$. They are applied to correct for residual differences in lepton selection efficiencies between data and simulation. The corresponding uncertainties are estimated to be about $3 \%$ per lepton for both flavors, and additionally $2 \%$ per lepton is assigned to the signal leptons due to the detector fast simulation. Assuming $100 \%$ correlation between the uncertainties on the corrections for the different leptons, a flat uncertainty of $9 \%$ is taken into account for the background, while $15 \%$ is considered for the signal. The uncertainty related to the HLT trigger efficiency is evaluated to amount to $3 \%$.

For the nonprompt and misidentified lepton background, several systematic uncertainties are considered. The statistical uncertainty from the application region, which is used 
to estimate this background contribution, ranges from 10 to $100 \%$. The regions where these uncertainties are large are generally regions where the overall contribution from this background is small. The uncertainty arising from the electroweak background subtraction in the measurement region for the tight-to-loose ratio is propagated from the uncertainty on the scale factor obtained from the fit to the control regions. In the case where no events are observed in the application region, an upper limit of the background expectation is used as determined from the upper limit at $68 \%$ confidence level (CL) multiplied by the most likely tight-to-loose ratio value.

The systematic uncertainty related to the extrapolation from the control regions to the signal regions for the nonprompt lepton background is estimated to be $30 \%$. This value has been extracted from closure tests performed by applying the method described in section 5 to simulated samples containing nonprompt leptons. From the simultaneous fit in the control regions, the uncertainty in the normalization of the WZ process is estimated

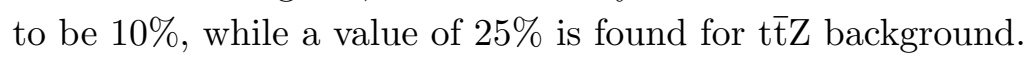

The limited size of the generated MC samples represents an additional source of un-

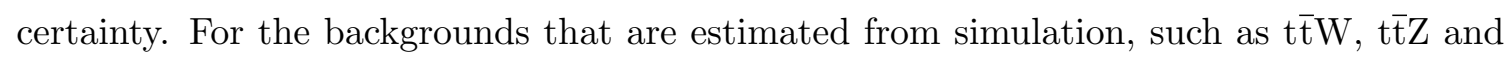
$\mathrm{t} \overline{\mathrm{t}} \mathrm{H}$, as well as for all the signal processes, this statistical uncertainty is computed from the number of MC events entering the signal regions and varies widely across the SRs.

For signal efficiency calculations additional uncertainties in the description of the initial-state radiation (ISR) are taken into account. The modeling of ISR by the version of the MADGRAPH5_aMC@NLO generator used for signal events was compared against a data sample of $t \bar{t}$ events in the dilepton final state. The corresponding corrections range from 0.51 to 0.92 , depending on the jet multiplicity. These corrections are then applied on simulated SUSY events based on the number of ISR jets to improve upon the MADGRAPH5_aMC@NLO modeling of the multiplicity of additional jets from ISR. Half the magnitude of these ISR corrections is assigned as an additional systematic uncertainty, which can be as large as $10 \%$.

The uncertainty in potential differences between the modeling of $p_{\mathrm{T}}^{\text {miss }}$ in data and the fast simulation arising from unclustered energy in the CMS detector is evaluated by comparing the reconstructed $p_{\mathrm{T}}^{\text {miss }}$ with the $p_{\mathrm{T}}^{\text {miss }}$ obtained using generator-level information. This uncertainty ranges up to $20 \%$.

Theoretical uncertainties include the uncertainty in the renormalization $\left(\mu_{\mathrm{R}}\right)$ and factorization $\left(\mu_{\mathrm{F}}\right)$ scales, and in the knowledge of the PDFs. These uncertainties are evaluated for several processes, namely $t \bar{t} \mathrm{~W}, \mathrm{t} \overline{\mathrm{t}} \mathrm{Z}$, and $\mathrm{t} \overline{\mathrm{t}} \mathrm{H}$, which are dominant backgrounds in several signal regions. Both the changes in the acceptance and cross sections related to these effects are taken into account and propagated to the final uncertainties.

For the study of the renormalization and factorization uncertainties, variations up and down by a factor of two with respect to the nominal values of $\mu_{\mathrm{F}}$ and $\mu_{\mathrm{R}}$ are evaluated. The maximum difference in the yields with respect to the nominal case is observed when both scales are varied up and down simultaneously. The effect on the overall cross section is found to be $\sim 13 \%$ for $\mathrm{t} \overline{\mathrm{t}} \mathrm{W}$ and $\sim 11 \%$ for $\mathrm{t} \overline{\mathrm{t}} \mathrm{H}$ backgrounds. The effect of the variations of $\mu_{\mathrm{F}}$ and $\mu_{\mathrm{R}}$ on the acceptance is taken as additional, uncorrelated uncertainty on the acceptance corresponding to different signal regions. This effect is found to vary between 
$3 \%$ and $18 \%$ depending on the SR and the process.

The uncertainty related to the PDFs is estimated from the 100 NNPDF 3.0 replicas, computing the deviation with respect to the nominal yield for each of them in every signal region (the cross section and acceptance effect are considered together) [60]. The rootmean-square of the variations is taken as the value of the systematic uncertainty. Since no significant differences between signal regions have been found, a flat uncertainty of $3 \%$ $(2 \%)$ is considered for $\mathrm{t} \overline{\mathrm{t} W}$ ( $\mathrm{t} \overline{\mathrm{t}} \mathrm{Z}$ and $\mathrm{t} \overline{\mathrm{t}} \mathrm{H}$ ) backgrounds. This value also includes the effect of the strong coupling constant variation, $\alpha_{\mathrm{S}}\left(M_{\mathrm{Z}}\right)$, which is added in quadrature. An extra, conservative, flat uncertainty of $50 \%$ is assigned to the yield of the remaining rare processes, which are not well measured.

\section{$7 \quad$ Results}

Comparisons between data and the predicted background of the distributions of the four event observables used for signal region categorization, namely $H_{\mathrm{T}}, p_{\mathrm{T}}^{\text {miss }}, M_{\mathrm{T}}$ and $N_{\mathrm{b} \text { jets }}$, as well as the lepton $p_{\mathrm{T}}$ spectra, the lepton flavor composition, and the event jet multiplicity are shown in figure 2 (figure 3 ) for events satisfying the selection criteria of the off-Z (on-Z). Figure 4 graphically presents a summary of the predicted background and observed event yields in the individual SR bins. The same information is also presented in tables 5 and 6 for the off- $Z$ and on- $Z$ regions, respectively. Table 7 represents the yields in the SSRs.

The number of events observed in data is found to be consistent with the predicted background yields in all 46 SRs. The results of the search are interpreted by setting limits on superpartner masses using simplified models. For each mass point, the observations, background predictions, and expected signal yields from all on- $\mathrm{Z}$ and off- $\mathrm{Z}$ search regions are combined to extract the minimum cross section that can be excluded at a 95\% CL using the $\mathrm{CL}_{\mathrm{s}}$ method [75-77], in which asymptotic approximations for the distribution of the test-statistic, which is a ratio of profiled likelihoods, are used [78]. Log-normal nuisance parameters are used to describe the uncertainties listed in section 6 .

The limits are shown in figure 5 for the T1tttt model (left) and for the T5qqqqVV model (right). In the T5qqqqVV model each gluino decays to a pair of light quarks and a neutralino $\left(\widetilde{\chi}_{2}^{0}\right)$ or chargino $\left(\widetilde{\chi}_{1}^{ \pm}\right)$, followed by the decay of that neutralino or chargino to a $\mathrm{W}$ or Z boson, respectively, and an LSP (figure 1, top right). The probability for the decay to proceed via the $\widetilde{\chi}_{1}^{+}, \widetilde{\chi}_{1}^{-}$, or $\widetilde{\chi}_{2}^{0}$ is taken to be $1 / 3$ for each case. In this scenario, the second neutralino $\widetilde{\chi}_{2}^{0}$ and chargino are assumed to be mass-degenerate, with masses equal to $0.5\left(m_{\widetilde{\mathrm{g}}}+m_{\widetilde{\chi}_{1}^{0}}\right)$.

The limits on the bottom squark pair production cross section are shown in figure 6 . In this model, the mass of the LSP is set to $50 \mathrm{GeV}$. Finally, the limits on the $\widetilde{\mathrm{t}}_{2}$ pair production cross section are shown in figure 7 . In this scenario, the mass difference between the $\widetilde{t}_{1}$ and the LSP is set to $175 \mathrm{GeV}$, the $\widetilde{\mathrm{t}}_{1}$ decays via a top quark to LSP, and the $\widetilde{\mathrm{t}}_{2}$ decays via a $\mathrm{Z}$ or Higgs boson to $\widetilde{\mathrm{t}}_{1}$. We consider the reference values $\mathcal{B}\left(\widetilde{\mathrm{t}}_{2} \rightarrow \widetilde{\mathrm{t}}_{1} \mathrm{Z}\right)=0$, 50 , and $100 \%$; the sensitivity is diminished for the $\widetilde{t}_{1} \mathrm{H}$ final state because of the additional branching factors for Higgs cascade decays to electrons or muons via gauge bosons or tau leptons. 

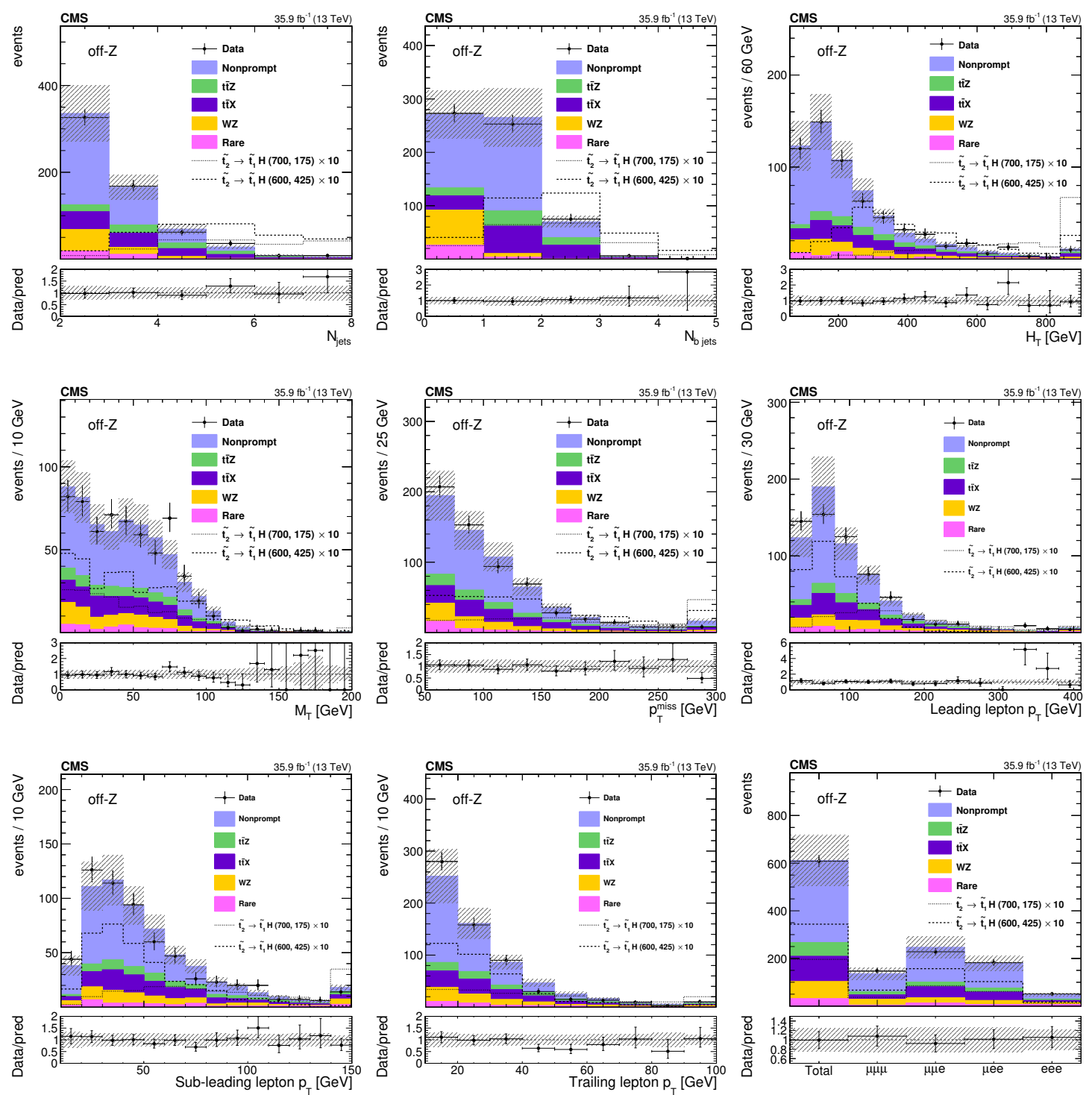

Figure 2. Background prediction and the observed event yields in the key observables for the off-Z baseline selection: the number of jets and b jets, $H_{\mathrm{T}}, M_{\mathrm{T}}, p_{\mathrm{T}}^{\text {miss }}$, the lepton $p_{\mathrm{T}}$ spectra and the event yields by flavor category are shown. The background events containing top quark(s) in association with a $\mathrm{W}, \mathrm{Z}$ or Higgs boson, except t $\mathrm{t} \overline{\mathrm{Z}}$, or another pair of top quarks are denoted as $\mathrm{t} \overline{\mathrm{t} X}$. The last bin includes the overflow events, and the hatched area represents the statistical and combined systematic uncertainties in the prediction. The lower panels show the ratio of the observed and predicted yields in each bin. For illustration the yields, multiplied by a factor 10, for two signal mass points in the T6ttHZ model, where the $\mathcal{B}\left(\widetilde{\mathrm{t}}_{2} \rightarrow \widetilde{\mathrm{t}}_{1} \mathrm{H}\right)=100 \%$, are displayed for non-compressed $\left(\mathrm{m}\left(\widetilde{\mathrm{t}}_{2}\right)=700 \mathrm{GeV}\right.$ and $\left.\mathrm{m}\left(\widetilde{\mathrm{t}}_{1}\right)=175 \mathrm{GeV}\right)$ and compressed $\left(\mathrm{m}\left(\widetilde{\mathrm{t}}_{2}\right)=600 \mathrm{GeV}\right.$ and $\left.\mathrm{m}\left(\widetilde{\mathrm{t}}_{1}\right)=425 \mathrm{GeV}\right)$ scenarios. 

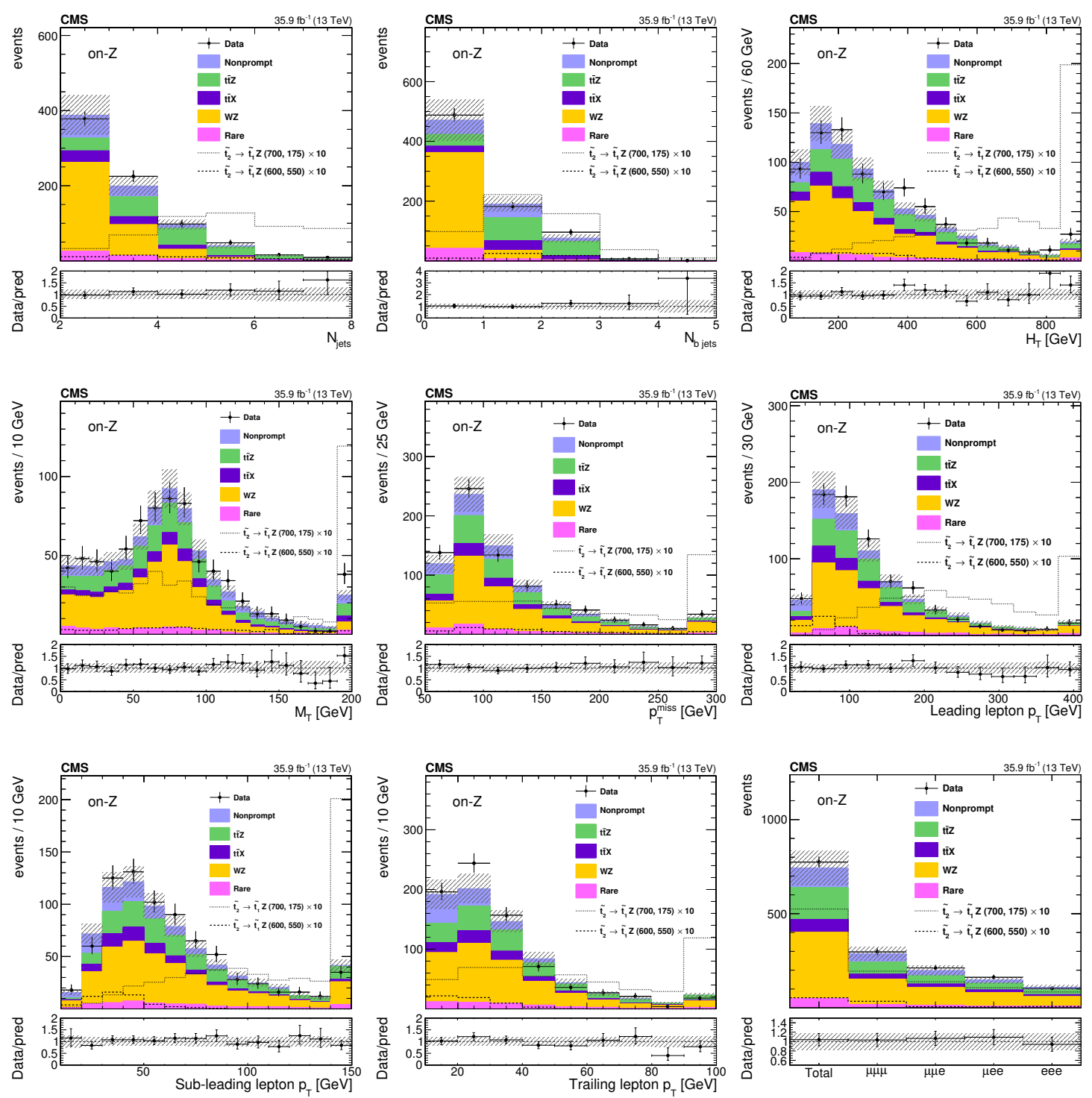

Figure 3. Background prediction and the observed event yields in the key observables of the on- $\mathrm{Z}$ baseline selection: the number of jets and b jets, $H_{\mathrm{T}}, M_{\mathrm{T}}, p_{\mathrm{T}}^{\text {miss }}$, the lepton $p_{\mathrm{T}}$ spectra and the event yields by flavor category are shown. The background events containing top quark(s) in association

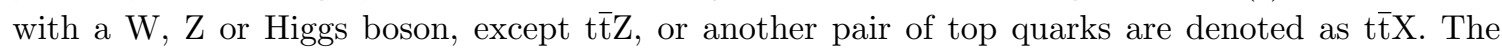
last bin includes the overflow events, and the hatched area represents the combined statistical and systematic uncertainties in the prediction. The lower panels show the ratio of the observed and predicted yields in each bin. For illustration the yields, multiplied by a factor 10, for two signal mass points in the T6ttHZ model, where the $\mathcal{B}\left(\widetilde{\mathrm{t}}_{2} \rightarrow \widetilde{\mathrm{t}}_{1} \mathrm{Z}\right)=100 \%$, are displayed for non-compressed $\left(\mathrm{m}\left(\widetilde{\mathrm{t}}_{2}\right)=700 \mathrm{GeV}\right.$ and $\left.\mathrm{m}\left(\widetilde{\mathrm{t}}_{1}\right)=175 \mathrm{GeV}\right)$ and compressed $\left(\mathrm{m}\left(\widetilde{\mathrm{t}}_{2}\right)=600 \mathrm{GeV}\right.$ and $\left.\mathrm{m}\left(\widetilde{\mathrm{t}}_{1}\right)=550 \mathrm{GeV}\right)$ scenarios. 

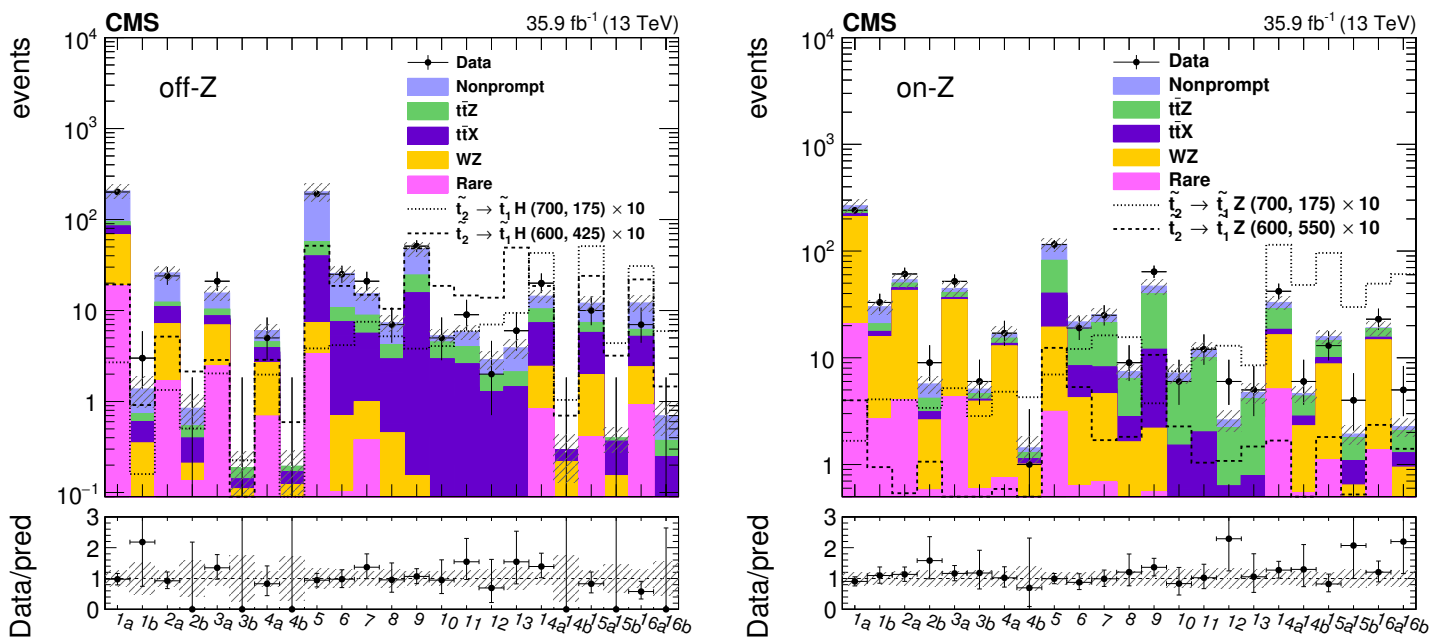

Figure 4. Background prediction and observed event yields in the 23 off- $\mathrm{Z}$ (left) and the 23 on- $\mathrm{Z}$ (right) signal regions. The background events containing top quark(s) in association with a W, Z

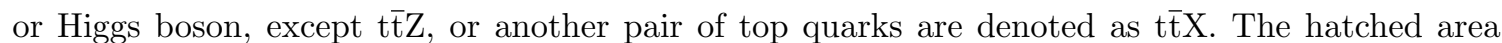
represents the statistical and systematic uncertainties on the prediction. The lower panels show the ratio of the observed and predicted yields in each bin. For illustration the yields, multiplied by a factor 10 , for $\widetilde{\mathrm{t}}_{2} \rightarrow \widetilde{\mathrm{t}}_{1} \mathrm{H}$ (left) and $\widetilde{\mathrm{t}}_{2} \rightarrow \widetilde{\mathrm{t}}_{1} \mathrm{Z}$ (right) decays are displayed for two signal mass points in the T6ttHZ model to represent compressed and non-compressed scenarios.

Search regions providing the best sensitivity to new physics scenarios depend on the considered models and their parameters. In the non-compressed scenario of the T1tttt model, the most sensitive region is off-Z SR16b (high $p_{\mathrm{T}}^{\text {miss }}$ and $M_{\mathrm{T}}$ region). When considering the compressed scenario, the contribution from SR16b region remains the largest, up to the most compressed cases where the SR12 off- $\mathrm{Z}$ region $\left(2 \mathrm{~b}\right.$ jets, medium $p_{\mathrm{T}}^{\text {miss }}$ and high $\left.H_{\mathrm{T}}\right)$ starts to contribute significantly. For the T5qqqqVV model in the non-compressed scenario, the most sensitive regions are on-Z SR16b and SR15b (high and medium $p_{\mathrm{T}}^{\text {miss }}$, high $H_{\mathrm{T}}$ and high $M_{\mathrm{T}}$ values). When moving towards more compressed scenarios, the most significant contributions come from the SR16b and SR15b on-Z regions, until reaching the compressed scenario where the most sensitive region is SR4b (medium $p_{\mathrm{T}}^{\mathrm{miss}}$, high $H_{\mathrm{T}}$ and high $M_{\mathrm{T}}$ ). The exclusion limit for T6ttWW model is dominated by both off-Z SR16 regions (high $p_{\mathrm{T}}^{\text {miss }}$ region). For the T6ttHZ model with $\mathcal{B}\left(\widetilde{\mathrm{t}}_{2} \rightarrow \widetilde{\mathrm{t}}_{1} \mathrm{Z}\right)=0 \%$, the limits in the non-compressed scenario are driven by the off-Z SR15a (high $H_{\mathrm{T}}$, medium $p_{\mathrm{T}}^{\text {miss }}$, low $M_{\mathrm{T}}$ ), while for compressed case by off-Z SR13 (high $N_{\mathrm{b} \text { jets }}$, low and medium $H_{\mathrm{T}}$ and $\left.p_{\mathrm{T}}^{\text {miss }}\right)$. For $\mathcal{B}\left(\widetilde{\mathrm{t}}_{2} \rightarrow \widetilde{\mathrm{t}}_{1} \mathrm{Z}\right)=50 \%$ in the non-compressed scenario, the on-Z SR16b region dominates the exclusion limit, while in the compressed scenario the on-Z SR13 (high $N_{\mathrm{b} \text { jets }}$ ) and SR15b (high $H_{\mathrm{T}}$, medium $p_{\mathrm{T}}^{\text {miss }}$, high $M_{\mathrm{T}}$ ) give the highest contribution. Finally, for $\mathcal{B}\left(\widetilde{\mathrm{t}}_{2} \rightarrow \widetilde{\mathrm{t}}_{1} \mathrm{Z}\right)=100 \%$ the on-Z SR16b plays the leading role in both compressed and non-compressed scenarios. 


\begin{tabular}{|c|c|c|c|c|c|c|}
\hline$N_{\mathrm{b}}$ jets & $H_{\mathrm{T}}[\mathrm{GeV}]$ & $p_{\mathrm{T}}^{\text {miss }}[\mathrm{GeV}]$ & $M_{\mathrm{T}}[\mathrm{GeV}]$ & Expected [events] & Observed [events] & $\mathrm{SR}$ \\
\hline \multirow{8}{*}{0} & \multirow{4}{*}{$60-400$} & \multirow{2}{*}{$50-150$} & $<120$ & $206 \pm 6 \pm 35$ & 201 & SR1a \\
\hline & & & $\geq 120$ & $1.4 \pm 0.5 \pm 0.2$ & 3 & SR1b \\
\hline & & \multirow{2}{*}{$150-300$} & $<120$ & $25.9 \pm 2.1 \pm 4.3$ & 24 & SR2a \\
\hline & & & $\geq 120$ & $0.84 \pm 0.34 \pm 0.12$ & 0 & $\mathrm{SR} 2 \mathrm{~b}$ \\
\hline & \multirow{4}{*}{$400-600$} & \multirow{2}{*}{$50-150$} & $<120$ & $15.6 \pm 1.6 \pm 2.1$ & 21 & SR3a \\
\hline & & & $\geq 120$ & $0.19 \pm 0.09 \pm 0.02$ & 0 & SR3b \\
\hline & & \multirow{2}{*}{$150-300$} & $<120$ & $6.0 \pm 0.8 \pm 0.7$ & 5 & SR4a \\
\hline & & & $\geq 120$ & $0.19 \pm 0.09 \pm 0.04$ & 0 & $\mathrm{SR} 4 \mathrm{~b}$ \\
\hline \multirow{4}{*}{1} & \multirow{2}{*}{$60-400$} & $50-150$ & \multirow{4}{*}{ Inclusive } & $202 \pm 6 \pm 44$ & 191 & SR5 \\
\hline & & $150-300$ & & $25.6 \pm 1.9 \pm 4.6$ & 25 & SR6 \\
\hline & \multirow{2}{*}{$400-600$} & $50-150$ & & $15.4 \pm 1.3 \pm 2.2$ & 21 & SR7 \\
\hline & & $150-300$ & & $7.3 \pm 1 \pm 1.1$ & 7 & SR8 \\
\hline \multirow{4}{*}{2} & \multirow{2}{*}{$60-400$} & $50-150$ & \multirow{4}{*}{ Inclusive } & $47.7 \pm 2.8 \pm 7.6$ & 51 & SR9 \\
\hline & & $150-300$ & & $5.3 \pm 0.5 \pm 0.6$ & 5 & SR10 \\
\hline & \multirow{2}{*}{$400-600$} & $50-150$ & & $5.8 \pm 0.7 \pm 0.8$ & 9 & SR11 \\
\hline & & $150-300$ & & $2.9 \pm 0.5 \pm 0.4$ & 2 & SR12 \\
\hline$\geq 3$ & $60-600$ & $50-300$ & Inclusive & $3.9 \pm 0.7 \pm 0.6$ & 6 & SR13 \\
\hline \multirow{6}{*}{ Inclusive } & \multirow{4}{*}{$\geq 600$} & \multirow{2}{*}{$50-150$} & $<120$ & $14.4 \pm 1.2 \pm 1.6$ & 20 & SR14a \\
\hline & & & $\geq 120$ & $0.28 \pm 0.14 \pm 0.04$ & 0 & SR14b \\
\hline & & \multirow{2}{*}{$150-300$} & $<120$ & $12.1 \pm 1.4 \pm 1.6$ & 10 & SR15a \\
\hline & & & $\geq 120$ & $0.40 \pm 0.12 \pm 0.05$ & 0 & SR15b \\
\hline & \multirow{2}{*}{$\geq 60$} & \multirow{2}{*}{$\geq 300$} & $<120$ & $12.1 \pm 1.5 \pm 1.9$ & 7 & SR16a \\
\hline & & & $\geq 120$ & $0.70 \pm 0.25 \pm 0.11$ & 0 & SR16b \\
\hline
\end{tabular}

Table 5. Expected and observed yields in the off-Z search regions. The first uncertainty states the statistical uncertainty, while the second represents the systematic uncertainty. 


\begin{tabular}{|c|c|c|c|c|c|c|}
\hline$N_{\mathrm{b} \text { jets }}$ & $H_{\mathrm{T}}[\mathrm{GeV}]$ & $p_{\mathrm{T}}^{\text {miss }}[\mathrm{GeV}]$ & $M_{\mathrm{T}}[\mathrm{GeV}]$ & Expected [events] & Observed [events] & $\mathrm{SR}$ \\
\hline \multirow{8}{*}{0} & \multirow{4}{*}{$60-400$} & \multirow{2}{*}{$70-150$} & $<120$ & $266 \pm 5 \pm 39$ & 241 & SR1a \\
\hline & & & $\geq 120$ & $30 \pm 2 \pm 4$ & 33 & SR1b \\
\hline & & \multirow{2}{*}{$150-300$} & $<120$ & $53.8 \pm 2.2 \pm 8$ & 61 & SR2a \\
\hline & & & $\geq 120$ & $5.7 \pm 0.8 \pm 0.7$ & 9 & $\mathrm{SR} 2 \mathrm{~b}$ \\
\hline & \multirow{4}{*}{$400-600$} & \multirow{2}{*}{$50-150$} & $<120$ & $44.6 \pm 1.9 \pm 6.5$ & 52 & SR3a \\
\hline & & & $\geq 120$ & $5.1 \pm 0.6 \pm 0.7$ & 6 & SR3b \\
\hline & & \multirow{2}{*}{$150-300$} & $<120$ & $16.6 \pm 1.3 \pm 2.5$ & 17 & SR4a \\
\hline & & & $\geq 120$ & $1.43 \pm 0.33 \pm 0.2$ & 1 & $\mathrm{SR} 4 \mathrm{~b}$ \\
\hline \multirow{4}{*}{1} & \multirow{2}{*}{$60-400$} & $70-150$ & \multirow{4}{*}{ Inclusive } & $116 \pm 4 \pm 15$ & 115 & SR5 \\
\hline & & $150-300$ & & $21.7 \pm 1.2 \pm 2.8$ & 19 & SR6 \\
\hline & \multirow{2}{*}{$400-600$} & $50-150$ & & $25.2 \pm 1.2 \pm 3.6$ & 25 & SR7 \\
\hline & & $150-300$ & & $7.5 \pm 0.8 \pm 1$ & 9 & SR8 \\
\hline \multirow{4}{*}{2} & \multirow{2}{*}{$60-400$} & $50-150$ & \multirow{4}{*}{ Inclusive } & $47 \pm 1.6 \pm 7.4$ & 64 & SR9 \\
\hline & & $150-300$ & & $7.2 \pm 0.8 \pm 1.2$ & 6 & SR10 \\
\hline & \multirow{2}{*}{$400-600$} & $50-150$ & & $11.7 \pm 1 \pm 2.1$ & 12 & SR11 \\
\hline & & $150-300$ & & $2.6 \pm 0.4 \pm 0.4$ & 6 & SR12 \\
\hline$\geq 3$ & $60-600$ & $50-300$ & Inclusive & $4.7 \pm 0.5 \pm 0.9$ & 5 & SR13 \\
\hline \multirow{6}{*}{ Inclusive } & \multirow{4}{*}{$\geq 600$} & \multirow{2}{*}{$50-150$} & $<120$ & $33 \pm 2 \pm 4$ & 42 & SR14a \\
\hline & & & $\geq 120$ & $4.6 \pm 0.6 \pm 0.6$ & 6 & SR14b \\
\hline & & \multirow{2}{*}{$150-300$} & $<120$ & $15.8 \pm 1.2 \pm 2$ & 13 & SR15a \\
\hline & & & $\geq 120$ & $1.9 \pm 0.3 \pm 0.2$ & 4 & SR15b \\
\hline & \multirow{2}{*}{$\geq 60$} & \multirow{2}{*}{$\geq 300$} & $<120$ & $19.1 \pm 1.1 \pm 2.8$ & 23 & SR16a \\
\hline & & & $\geq 120$ & $2.28 \pm 0.35 \pm 0.26$ & 5 & SR16b \\
\hline
\end{tabular}

Table 6. Expected and observed yields in the on-Z search regions. The first uncertainty states the statistical uncertainty, while the second represents the systematic uncertainty.

\begin{tabular}{|l|c|c|c|c|}
\hline & SSR1 & SSR2 & SSR3 & SSR4 \\
\hline Nonprompt & $0.63 \pm 0.38 \pm 0.19$ & $0.00 \pm 0.00_{-0.0}^{+0.3}$ & $0.46 \pm 0.37 \pm 0.14$ & $0.21_{-0.21}^{+0.23} \pm 0.06$ \\
$\mathrm{t} \overline{\mathrm{tZ}}$ & $0.14 \pm 0.06 \pm 0.03$ & $0.05 \pm 0.03 \pm 0.01$ & $1.27 \pm 0.18 \pm 0.31$ & $0.54 \pm 0.10 \pm 0.13$ \\
$\mathrm{t} \mathrm{t} X$ & $0.23 \pm 0.04 \pm 0.05$ & $0.11 \pm 0.04 \pm 0.02$ & $0.50 \pm 0.07 \pm 0.08$ & $0.17 \pm 0.03 \pm 0.02$ \\
WZ & $0.01 \pm 0.01 \pm 0.01$ & $0.01 \pm 0.01 \pm 0.01$ & $1.03 \pm 0.28 \pm 0.21$ & $0.01 \pm 0.01 \pm 0.01$ \\
Rare & $0.12 \pm 0.06 \pm 0.05$ & $0.01 \pm 0.01 \pm 0.01$ & $0.40 \pm 0.09 \pm 0.14$ & $0.01 \pm 0.01 \pm 0.01$ \\
\hline Total & $1.1 \pm 0.4 \pm 0.2$ & $0.18 \pm 0.05_{-0.02}^{+0.3}$ & $3.7 \pm 0.5 \pm 0.4$ & $0.94_{-0.23}^{+0.26} \pm 0.15$ \\
\hline Observed & 0 & 0 & 6 & 2 \\
\hline
\end{tabular}

Table 7. Expected and observed yields in the super signal regions. The background events con-

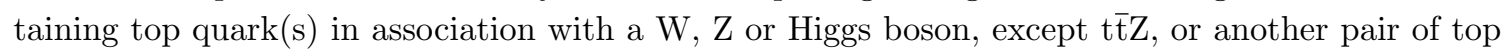

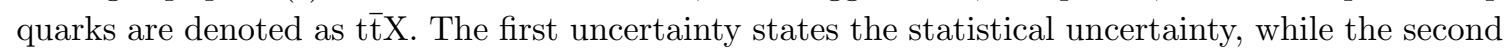
represents the systematic uncertainty. 

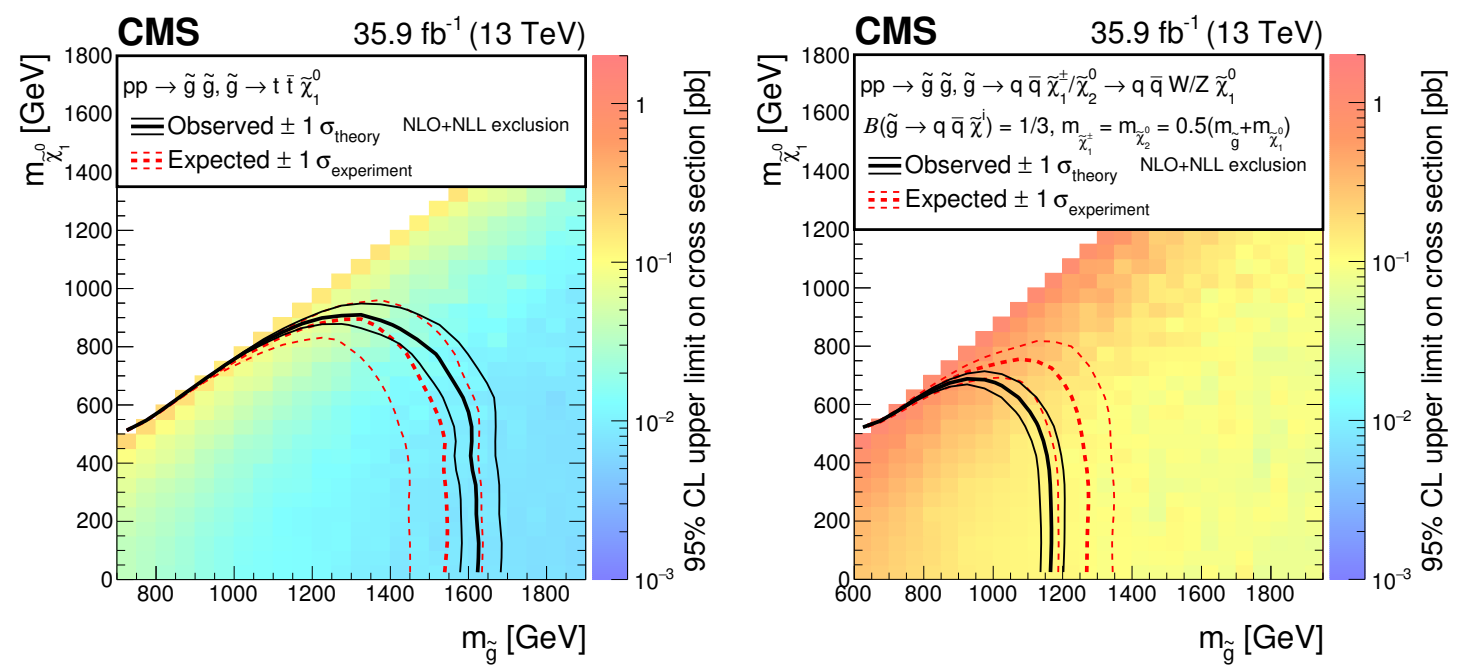

Figure 5. Cross section upper limits at $95 \%$ CL in the $m_{\widetilde{\chi}_{1}^{0}}$ versus $m_{\widetilde{\mathrm{g}}}$ plane for T1tttt (left) and T5qqqqVV (right) simplified models. For the latter model the branching fraction of gluino decay to neutralino or chargino is equal to $1 / 3$ and $m_{\tilde{\chi}_{1}^{ \pm}}=m_{\tilde{\chi}_{2}^{0}}=0.5\left(m_{\widetilde{\mathrm{g}}}+m_{\tilde{\chi}_{1}^{0}}\right)$. The excluded regions are to the left and below the observed and expected limit curves. The color scale indicates the excluded cross section at a given point in the mass plane.

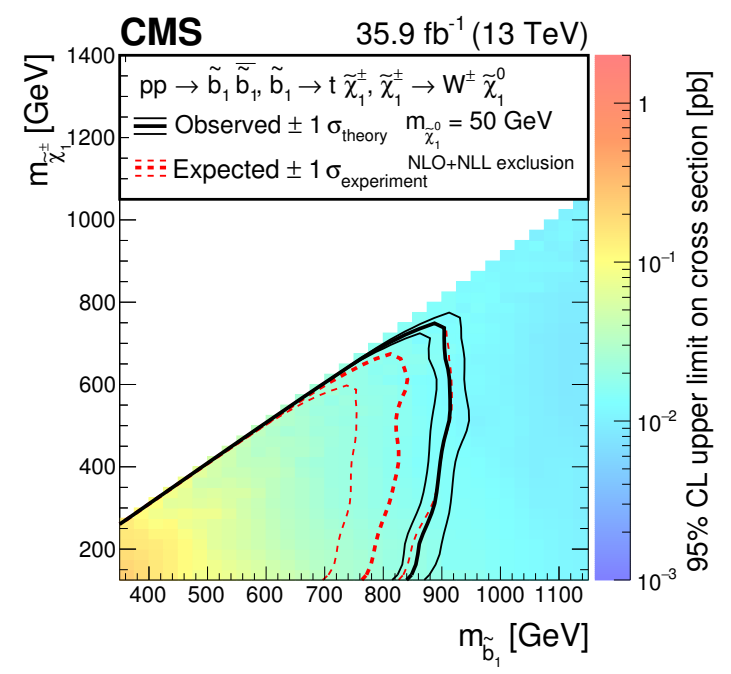

Figure 6. Cross section upper limits at $95 \% \mathrm{CL}$ in the $m_{\widetilde{\chi}_{1}^{ \pm}}$versus $m_{\widetilde{\mathrm{b}}_{1}}$ plane for T6ttWW simplified model. The mass of the neutralino is set to $50 \mathrm{GeV}$. The descriptions of the excluded regions and color scale are the same as in figure 5 . 

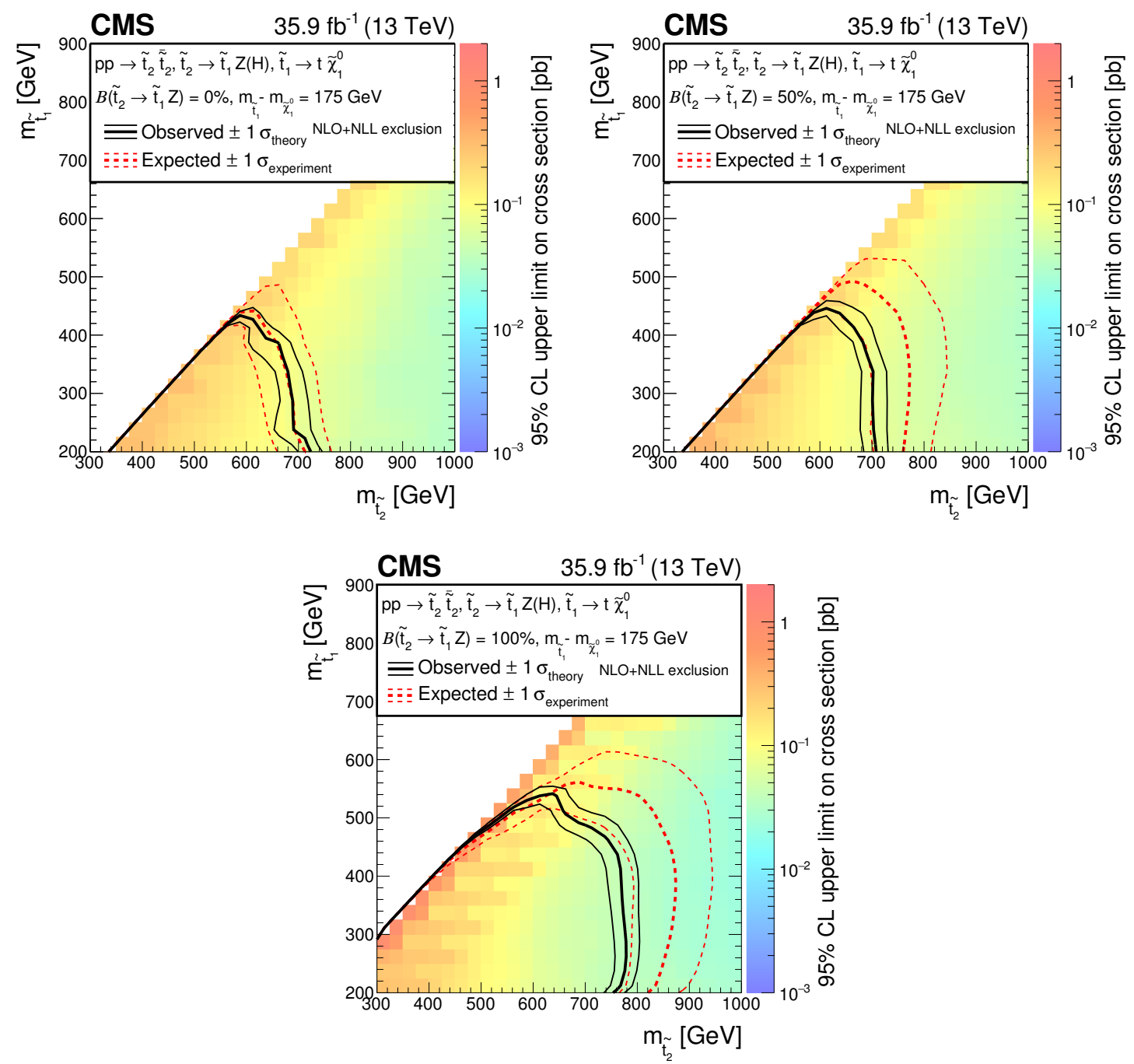

Figure 7. Cross section upper limits at $95 \%$ CL in the $m_{\widetilde{\mathrm{t}}_{1}}$ versus $m_{\mathfrak{\mathrm { t }}_{2}}$ plane for T6ttHZ simplified model. Different branching fractions of the decay $\widetilde{\mathrm{t}}_{2} \rightarrow \widetilde{\mathrm{t}}_{1} \mathrm{Z}$ are considered: $0 \%$ (top left), $50 \%$ (top right), and $100 \%$ (bottom). The mass difference between the lighter top squark $\left(\widetilde{\mathrm{t}}_{1}\right)$ and a neutralino is close to the mass of the top quark. The descriptions of the excluded regions and color scale are the same as in figure 5 .

\section{Conclusions}

A search for physics beyond the standard model in final states with at least three electrons or muons in any combination, jets, and missing transverse momentum has been presented using data collected by the CMS detector in 2016 at $\sqrt{s}=13 \mathrm{TeV}$, corresponding to an integrated luminosity of $35.9 \mathrm{fb}^{-1}$. The analysis makes use of control regions in data to estimate reducible backgrounds and to validate simulations used to estimate irreducible background processes. To maximize sensitivity to a broad range of possible signal models, 46 exclusive signal regions are defined. No significant deviation from the expected standard model background is observed in any of these signal regions. 
The results are interpreted using a simplified gluino-pair production model that features cascade decays producing four top quarks and two neutralinos. In this model, gluinos with a mass up to $1610 \mathrm{GeV}$ are excluded in the case of a massless LSP. The maximum excluded LSP mass is $900 \mathrm{GeV}$. This represents an improvement of approximately 435 and $250 \mathrm{GeV}$, respectively, compared to the exclusion limit set in a similar search based on data collected with the CMS detector in 2015, corresponding to an integrated luminosity of $2.3 \mathrm{fb}^{-1}[39]$.

For the simplified model of gluino-gluino production with decay to light-flavor quark jets, two vector bosons and neutralinos, gluino masses up to $1160 \mathrm{GeV}$ and neutralino masses up to $680 \mathrm{GeV}$ can be excluded. The limit on gluino and neutralino masses extends the corresponding limit from the previous analysis by about 335 and $180 \mathrm{GeV}$, respectively.

For a simplified model of bottom squark pair production decaying to top quarks, W bosons and neutralinos, bottom squark masses up to $840 \mathrm{GeV}$ are excluded for a low mass chargino, while chargino masses are excluded up to $750 \mathrm{GeV}$. These extend the previous limits by $380 \mathrm{GeV}$ for each particle.

Finally, for a simplified heavy top squark pair production model with further decays to two top quarks, Higgs or Z bosons, and neutralinos, the $\widetilde{t}_{2}$ mass is excluded up to 720, 780, and $710 \mathrm{GeV}$ for models with an exclusive $\widetilde{t}_{2} \rightarrow \widetilde{\mathrm{t}}_{1} \mathrm{H}$ decay, an exclusive $\widetilde{\mathrm{t}}_{2} \rightarrow \widetilde{\mathrm{t}}_{1} \mathrm{Z}$ decay, and an equally probable mix of those two decays, while the $\widetilde{t}_{1}$ mass is excluded up to 430 , 540 , and $450 \mathrm{GeV}$ for the same branching fractions. This significantly improves the results obtained with the $8 \mathrm{TeV}$ dataset [36].

\section{Acknowledgments}

We congratulate our colleagues in the CERN accelerator departments for the excellent performance of the LHC and thank the technical and administrative staffs at CERN and at other CMS institutes for their contributions to the success of the CMS effort. In addition, we gratefully acknowledge the computing centres and personnel of the Worldwide LHC Computing Grid for delivering so effectively the computing infrastructure essential to our analyses. Finally, we acknowledge the enduring support for the construction and operation of the LHC and the CMS detector provided by the following funding agencies: BMWFW and FWF (Austria); FNRS and FWO (Belgium); CNPq, CAPES, FAPERJ, and FAPESP (Brazil); MES (Bulgaria); CERN; CAS, MoST, and NSFC (China); COLCIENCIAS (Colombia); MSES and CSF (Croatia); RPF (Cyprus); SENESCYT (Ecuador); MoER, ERC IUT, and ERDF (Estonia); Academy of Finland, MEC, and HIP (Finland); CEA and CNRS/IN2P3 (France); BMBF, DFG, and HGF (Germany); GSRT (Greece); OTKA and NIH (Hungary); DAE and DST (India); IPM (Iran); SFI (Ireland); INFN (Italy); MSIP and NRF (Republic of Korea); LAS (Lithuania); MOE and UM (Malaysia); BUAP, CINVESTAV, CONACYT, LNS, SEP, and UASLP-FAI (Mexico); MBIE (New Zealand); PAEC (Pakistan); MSHE and NSC (Poland); FCT (Portugal); JINR (Dubna); MON, RosAtom, RAS, RFBR and RAEP (Russia); MESTD (Serbia); SEIDI, CPAN, PCTI and FEDER (Spain); Swiss Funding Agencies (Switzerland); MST (Taipei); ThEPCenter, 
IPST, STAR, and NSTDA (Thailand); TUBITAK and TAEK (Turkey); NASU and SFFR (Ukraine); STFC (United Kingdom); DOE and NSF (U.S.A.).

Individuals have received support from the Marie-Curie programme and the European Research Council and Horizon 2020 Grant, contract No. 675440 (European Union); the Leventis Foundation; the A. P. Sloan Foundation; the Alexander von Humboldt Foundation; the Belgian Federal Science Policy Office; the Fonds pour la Formation à la Recherche dans l'Industrie et dans l'Agriculture (FRIA-Belgium); the Agentschap voor Innovatie door Wetenschap en Technologie (IWT-Belgium); the Ministry of Education, Youth and Sports (MEYS) of the Czech Republic; the Council of Science and Industrial Research, India; the HOMING PLUS programme of the Foundation for Polish Science, cofinanced from European Union, Regional Development Fund, the Mobility Plus programme of the Ministry of Science and Higher Education, the National Science Center (Poland), contracts Harmonia 2014/14/M/ST2/00428, Opus 2014/13/B/ST2/02543, 2014/15/B/ST2/03998, and 2015/19/B/ST2/02861, Sonata-bis 2012/07/E/ST2/01406; the National Priorities Research Program by Qatar National Research Fund; the Programa Severo Ochoa del Principado de Asturias; the Thalis and Aristeia programmes cofinanced by EU-ESF and the Greek NSRF; the Rachadapisek Sompot Fund for Postdoctoral Fellowship, Chulalongkorn University and the Chulalongkorn Academic into Its 2nd Century Project Advancement Project (Thailand); the Welch Foundation, contract C-1845; and the Weston Havens Foundation (U.S.A.).

Open Access. This article is distributed under the terms of the Creative Commons Attribution License (CC-BY 4.0), which permits any use, distribution and reproduction in any medium, provided the original author(s) and source are credited.

\section{References}

[1] O.J.P. Eboli, R. Zukanovich Funchal and T.L. Lungov, Multilepton signatures for leptoquarks, Phys. Rev. D 59 (1999) 035002 [hep-ph/9808288] [INSPIRE].

[2] N. Craig et al., Searching for $t \rightarrow$ ch with multi-leptons, Phys. Rev. D 86 (2012) 075002 [arXiv: 1207.6794] [INSPIRE].

[3] N. Craig, J. Hajer, Y.-Y. Li, T. Liu and H. Zhang, Heavy Higgs bosons at low $\tan \beta$ : from the LHC to $100 \mathrm{TeV}$, JHEP 01 (2017) 018 [arXiv:1605.08744] [INSPIRE].

[4] N. Craig et al., Multi-lepton signals of multiple Higgs bosons, JHEP 02 (2013) 033 [arXiv: 1210.0559] [INSPIRE].

[5] C.-S. Chen and Y.-J. Zheng, LHC signatures for the cascade seesaw mechanism, PTEP 2015 (2015) 103B02 [arXiv:1312.7207] [INSPIRE].

[6] A. Lazopoulos, T. McElmurry, K. Melnikov and F. Petriello, Next-to-leading order QCD corrections to $t \bar{t} Z$ production at the LHC, Phys. Lett. B 666 (2008) 62 [arXiv:0804.2220] [INSPIRE].

[7] A. Kardos, Z. Trócsányi and C. Papadopoulos, Top quark pair production in association with a Z-boson at NLO accuracy, Phys. Rev. D 85 (2012) 054015 [arXiv:1111.0610] [InSPIRE]. 
[8] J.M. Campbell and R.K. Ellis, $t \bar{t} W^{+-}$production and decay at NLO, JHEP 07 (2012) 052 [arXiv: 1204.5678] [INSPIRE].

[9] J. Campbell, R.K. Ellis and R. Röntsch, Single top production in association with a $Z$ boson at the LHC, Phys. Rev. D 87 (2013) 114006 [arXiv:1302.3856] [InSPIRE].

[10] A. Kulesza, L. Motyka, T. Stebel and V. Theeuwes, Soft gluon resummation for associated $t \bar{t} H$ production at the LHC, JHEP 03 (2016) 065 [arXiv:1509.02780] [INSPIRE].

[11] A. Broggio et al., Associated production of a top pair and a Higgs boson beyond NLO, JHEP 03 (2016) 124 [arXiv: 1510.01914] [INSPIRE].

[12] M. Grazzini, S. Kallweit, D. Rathlev and M. Wiesemann, $W^{ \pm} Z$ production at the LHC: fiducial cross sections and distributions in NNLO QCD, JHEP 05 (2017) 139 [arXiv: 1703.09065] [INSPIRE].

[13] F. Cascioli et al., ZZ production at hadron colliders in NNLO QCD, Phys. Lett. B 735 (2014) 311 [arXiv:1405.2219] [INSPIRE].

[14] F. Caola, K. Melnikov, R. Röntsch and L. Tancredi, QCD corrections to ZZ production in gluon fusion at the LHC, Phys. Rev. D 92 (2015) 094028 [arXiv: 1509.06734] [InSPIRE].

[15] J.M. Campbell, R.K. Ellis, M. Czakon and S. Kirchner, Two loop correction to interference in $g g \rightarrow Z Z$, JHEP 08 (2016) 011 [arXiv: 1605.01380] [INSPIRE].

[16] T. Binoth, G. Ossola, C.G. Papadopoulos and R. Pittau, NLO QCD corrections to tri-boson production, JHEP 06 (2008) 082 [arXiv: 0804.0350] [INSPIRE].

[17] D.T. Nhung, L.D. Ninh and M.M. Weber, NLO corrections to WWZ production at the LHC, JHEP 12 (2013) 096 [arXiv: 1307.7403] [INSPIRE].

[18] S. Yong-Bai et al., $N L O Q C D+N L O E W$ corrections to $W Z Z$ productions with leptonic decays at the LHC, JHEP 10 (2015) 186 [Erratum ibid. 10 (2016) 156] [arXiv:1507.03693] [INSPIRE].

[19] Y.-B. Shen et al., NLO QCD and electroweak corrections to $W W W$ production at the $L H C$, Phys. Rev. D 95 (2017) 073005 [arXiv: 1605.00554] [INSPIRE].

[20] W. Hong et al., NLO QCD $+E W$ corrections to ZZZ production with subsequent leptonic decays at the LHC, J. Phys. G 43 (2016) 115001 [arXiv:1610.05876] [INSPIRE].

[21] ATLAS collaboration, Measurement of the $t \bar{t} Z$ and $t \bar{t} W$ production cross sections in multilepton final states using $3.2 \mathrm{fb}^{-1}$ of pp collisions at $\sqrt{s}=13$ TeV with the ATLAS detector, Eur. Phys. J. C 77 (2017) 40 [arXiv:1609.01599] [InSPIRE].

[22] ATLAS collaboration, Measurement of the $W^{ \pm} Z$ boson pair-production cross section in pp collisions at $\sqrt{s}=13 \mathrm{TeV}$ with the ATLAS Detector, Phys. Lett. B 762 (2016) 1 [arXiv: 1606.04017] [INSPIRE].

[23] CMS collaboration, Measurement of the WZ production cross section in pp collisions at $\sqrt{s}=13 \mathrm{TeV}$, Phys. Lett. B 766 (2017) 268 [arXiv:1607.06943] [INSPIRE].

[24] ATLAS collaboration, Measurement of the $Z Z$ production cross section in PP collisions at $\sqrt{s}=13$ TeV with the ATLAS detector, Phys. Rev. Lett. 116 (2016) 101801 [arXiv: 1512.05314] [INSPIRE].

[25] CMS collaboration, Measurement of the ZZ production cross section and $Z \rightarrow \ell^{+} \ell^{-} \ell^{\prime+} \ell^{\prime-}$ branching fraction in pp collisions at $\sqrt{s}=13 \mathrm{TeV}$, Phys. Lett. B 763 (2016) 280 [Erratum ibid. B 772 (2017) 884] [arXiv: 1607.08834] [INSPIRE]. 
[26] P. Ramond, Dual theory for free fermions, Phys. Rev. D 3 (1971) 2415 [inSPIRE].

[27] Yu. A. Golfand and E.P. Likhtman, Extension of the algebra of Poincaré group generators and violation of $p$ invariance, JETP Lett. 13 (1971) 323 [Pisma Zh. Eksp. Teor. Fiz. 13 (1971) 452] [INSPIRE].

[28] A. Neveu and J.H. Schwarz, Factorizable dual model of pions, Nucl. Phys. B 31 (1971) 86 [INSPIRE].

[29] D.V. Volkov and V.P. Akulov, Possible universal neutrino interaction, JETP Lett. 16 (1972) 438 [INSPIRE].

[30] J. Wess and B. Zumino, A lagrangian model invariant under supergauge transformations, Phys. Lett. 49B (1974) 52 [INSPIRE].

[31] J. Wess and B. Zumino, Supergauge transformations in four-dimensions, Nucl. Phys. B 70 (1974) 39 [INSPIRE].

[32] P. Fayet, Supergauge invariant extension of the Higgs mechanism and a model for the electron and its neutrino, Nucl. Phys. B 90 (1975) 104 [inSPIRE].

[33] H.P. Nilles, Supersymmetry, supergravity and particle physics, Phys. Rept. 110 (1984) 1 [INSPIRE].

[34] S.P. Martin, A Supersymmetry primer, Adv. Ser. Direct. High Energy Phys. 21 (2010) 1 [hep-ph/9709356] [INSPIRE].

[35] LHC New Physics Working Group collaboration, D. Alves, Simplified models for LHC new physics searches, J. Phys. G 39 (2012) 105005 [arXiv: 1105.2838] [INSPIRE].

[36] CMS collaboration, Search for top-squark pairs decaying into Higgs or $Z$ bosons in pp collisions at $\sqrt{s}=8 \mathrm{TeV}$, Phys. Lett. B 736 (2014) 371 [arXiv:1405.3886] [inSPIRE].

[37] ATLAS collaboration, Search for direct top squark pair production in events with a Higgs or $Z$ boson and missing transverse momentum in $\sqrt{s}=13 \mathrm{TeV}$ pp collisions with the ATLAS detector, JHEP 08 (2017) 006 [arXiv:1706.03986] [INSPIRE].

[38] ATLAS collaboration, Search for supersymmetry in final states with two same-sign or three leptons and jets using $36 \mathrm{fb}^{-1}$ of $\sqrt{\mathrm{s}}=13 \mathrm{TeV}$ pp collision data with the ATLAS detector, JHEP 09 (2017) 084 [arXiv: 1706.03731] [INSPIRE].

[39] CMS collaboration, Search for new phenomena with multiple charged leptons in proton-proton collisions at $\sqrt{s}=13 \mathrm{TeV}$, Eur. Phys. J. C 77 (2017) 635 [arXiv: 1701.06940] [INSPIRE].

[40] CMS collaboration, Performance of photon reconstruction and identification with the CMS detector in proton-proton collisions at $\sqrt{s}=8 \mathrm{TeV}, 2015$ JINST $10 \mathrm{P} 08010$ [arXiv: 1502.02702] [INSPIRE].

[41] CMS collaboration, Performance of CMS muon reconstruction in pp collision events at $\sqrt{s}=7 \mathrm{TeV}, 2012$ JINST 7 P10002 [arXiv:1206.4071] [INSPIRE].

[42] CMS collaboration, The CMS trigger system, 2017 JINST 12 P01020 [arXiv:1609.02366] [INSPIRE].

[43] CMS collaboration, The CMS experiment at the CERN LHC, 2008 JINST 3 S08004 [INSPIRE].

[44] CMS collaboration, Particle-flow reconstruction and global event description with the CMS detector, 2017 JINST 12 P10003 [arXiv:1706. 04965] [INSPIRE]. 
[45] M. Cacciari, G.P. Salam and G. Soyez, The anti- $k_{t}$ jet clustering algorithm, JHEP 04 (2008) 063 [arXiv: 0802.1189] [INSPIRE].

[46] M. Cacciari, G.P. Salam and G. Soyez, FastJet user manual, Eur. Phys. J. C 72 (2012) 1896 [arXiv:1111.6097] [INSPIRE].

[47] CMS collaboration, Determination of jet energy calibration and transverse momentum resolution in CMS, 2011 JINST 6 P11002 [arXiv:1107.4277] [INSPIRE].

[48] CMS collaboration, Jet energy scale and resolution in the CMS experiment in pp collisions at $8 \mathrm{TeV}, 2017$ JINST $12 \mathrm{P} 02014$ [arXiv: 1607.03663] [INSPIRE].

[49] CMS collaboration, Identification of b-quark jets with the CMS experiment, 2013 JINST 8 P04013 [arXiv: 1211.4462] [INSPIRE].

[50] CMS collaboration, Identification of heavy-flavour jets with the CMS detector in pp collisions at $13 \mathrm{TeV}$, arXiv:1712.07158 [INSPIRE].

[51] CMS collaboration, Performance of the CMS missing transverse momentum reconstruction in pp data at $\sqrt{s}=8 \mathrm{TeV}, 2015$ JINST $10 \mathrm{P} 02006$ [arXiv:1411.0511] [INSPIRE].

[52] CMS collaboration, Performance of missing energy reconstruction in $13 \mathrm{TeV}$ pp collision data using the CMS detector, CMS-PAS-JME-16-004 (2016).

[53] CMS collaboration, Performance of electron reconstruction and selection with the CMS detector in proton-proton collisions at $\sqrt{s}=8 \mathrm{TeV}, 2015$ JINST $10 \mathrm{P} 06005$ [arXiv: 1502.02701] [INSPIRE].

[54] CMS collaboration, Search for new physics in same-sign dilepton events in proton-proton collisions at $\sqrt{s}=13 \mathrm{TeV}$, Eur. Phys. J. C 76 (2016) 439 [arXiv:1605.03171] [InSPIRE].

[55] J. Alwall et al., The automated computation of tree-level and next-to-leading order differential cross sections and their matching to parton shower simulations, JHEP 07 (2014) 079 [arXiv: 1405.0301] [INSPIRE].

[56] S. Alioli, P. Nason, C. Oleari and E. Re, A general framework for implementing NLO calculations in shower Monte Carlo programs: the POWHEG BOX, JHEP 06 (2010) 043 [arXiv: 1002.2581] [INSPIRE].

[57] H.B. Hartanto, B. Jager, L. Reina and D. Wackeroth, Higgs boson production in association with top quarks in the POWHEG BOX, Phys. Rev. D 91 (2015) 094003 [arXiv:1501.04498] [INSPIRE].

[58] T. Melia, P. Nason, R. Rontsch and G. Zanderighi, $W^{+} W^{-}, W Z$ and $Z Z$ production in the POWHEG BOX, JHEP 11 (2011) 078 [arXiv:1107.5051] [INSPIRE].

[59] P. Nason and G. Zanderighi, $W^{+} W^{-}, W Z$ and $Z Z$ production in the POWHEG-BOX-V2, Eur. Phys. J. C 74 (2014) 2702 [arXiv:1311.1365] [INSPIRE].

[60] NNPDF collaboration, R.D. Ball et al., Parton distributions for the LHC Run II, JHEP 04 (2015) 040 [arXiv: 1410.8849] [inSPIRE].

[61] T. Sjöstrand, S. Mrenna and P.Z. Skands, A brief introduction to PYTHIA 8.1, Comput. Phys. Commun. 178 (2008) 852 [arXiv:0710.3820] [INSPIRE].

[62] P. Skands, S. Carrazza and J. Rojo, Tuning PYTHIA 8.1: the Monash 2013 Tune, Eur. Phys. J. C 74 (2014) 3024 [arXiv: 1404.5630] [InSPIRE].

[63] CMS collaboration, Event generator tunes obtained from underlying event and multiparton scattering measurements, Eur. Phys. J. C 76 (2016) 155 [arXiv:1512.00815] [INSPIRE]. 
[64] J. Alwall et al., Comparative study of various algorithms for the merging of parton showers and matrix elements in hadronic collisions, Eur. Phys. J. C 53 (2008) 473 [arXiv:0706.2569] [INSPIRE].

[65] R. Frederix and S. Frixione, Merging meets matching in MC@NLO, JHEP 12 (2012) 061 [arXiv: 1209.6215] [INSPIRE].

[66] GEANT4 collaboration, S. Agostinelli et al., GEANT4 - a simulation toolkit, Nucl. Instrum. Meth. A 506 (2003) 250 [INSPIRE].

[67] CMS collaboration, The fast simulation of the CMS detector at LHC, J. Phys. Conf. Ser. 331 (2011) 032049 [INSPIRE].

[68] W. Beenakker, R. Hopker, M. Spira and P.M. Zerwas, Squark and gluino production at hadron colliders, Nucl. Phys. B 492 (1997) 51 [hep-ph/9610490] [INSPIRE].

[69] A. Kulesza and L. Motyka, Threshold resummation for squark-antisquark and gluino-pair production at the LHC, Phys. Rev. Lett. 102 (2009) 111802 [arXiv:0807.2405] [INSPIRE].

[70] A. Kulesza and L. Motyka, Soft gluon resummation for the production of gluino-gluino and squark-antisquark pairs at the LHC, Phys. Rev. D 80 (2009) 095004 [arXiv: 0905.4749] [INSPIRE].

[71] W. Beenakker et al., Soft-gluon resummation for squark and gluino hadroproduction, JHEP 12 (2009) 041 [arXiv: 0909.4418] [INSPIRE].

[72] W. Beenakker et al., Squark and gluino hadroproduction, Int. J. Mod. Phys. A 26 (2011) 2637 [arXiv: 1105.1110] [INSPIRE].

[73] C. Borschensky et al., Squark and gluino production cross sections in pp collisions at $\sqrt{s}=13,14,33$ and 100 TeV, Eur. Phys. J. C 74 (2014) 3174 [arXiv:1407.5066] [InSPIRE].

[74] Particle Data Group collaboration, C. Patrignani et al., Review of particle physics, Chin. Phys. C 40 (2016) 100001 [INSPIRE].

[75] T. Junk, Confidence level computation for combining searches with small statistics, Nucl. Instrum. Meth. A 434 (1999) 435 [hep-ex/9902006] [INSPIRE].

[76] A.L. Read, Presentation of search results: the $C L_{s}$ technique, J. Phys. G 28 (2002) 2693 [INSPIRE].

[77] ATLAS and CMS collaborations and The LHC Higgs Combination Group, Procedure for the LHC Higgs boson search combination in Summer 2011, ATL-PHYS-PUB-2011-11 (2011).

[78] G. Cowan, K. Cranmer, E. Gross and O. Vitells, Asymptotic formulae for likelihood-based tests of new physics, Eur. Phys. J. C 71 (2011) 1554 [Erratum ibid. C 73 (2013) 2501] [arXiv: 1007.1727] [INSPIRE]. 


\section{The CMS collaboration}

\section{Yerevan Physics Institute, Yerevan, Armenia}

A.M. Sirunyan, A. Tumasyan

\section{Institut für Hochenergiephysik, Wien, Austria}

W. Adam, F. Ambrogi, E. Asilar, T. Bergauer, J. Brandstetter, E. Brondolin, M. Dragicevic, J. Erö, M. Flechl, M. Friedl, R. Frühwirth ${ }^{1}$, V.M. Ghete, J. Grossmann, J. Hrubec, M. Jeitler ${ }^{1}$, A. König, N. Krammer, I. Krätschmer, D. Liko, T. Madlener, I. Mikulec, E. Pree, D. Rabady, N. Rad, H. Rohringer, J. Schieck ${ }^{1}$, R. Schöfbeck, M. Spanring, D. Spitzbart, W. Waltenberger, J. Wittmann, C.-E. Wulz ${ }^{1}$, M. Zarucki

Institute for Nuclear Problems, Minsk, Belarus

V. Chekhovsky, V. Mossolov, J. Suarez Gonzalez

\section{Universiteit Antwerpen, Antwerpen, Belgium}

E.A. De Wolf, D. Di Croce, X. Janssen, J. Lauwers, M. Van De Klundert, H. Van Haevermaet, P. Van Mechelen, N. Van Remortel

\section{Vrije Universiteit Brussel, Brussel, Belgium}

S. Abu Zeid, F. Blekman, J. D’Hondt, I. De Bruyn, J. De Clercq, K. Deroover, G. Flouris, D. Lontkovskyi, S. Lowette, S. Moortgat, L. Moreels, Q. Python, K. Skovpen, S. Tavernier, W. Van Doninck, P. Van Mulders, I. Van Parijs

\section{Université Libre de Bruxelles, Bruxelles, Belgium}

D. Beghin, H. Brun, B. Clerbaux, G. De Lentdecker, H. Delannoy, G. Fasanella, L. Favart, R. Goldouzian, A. Grebenyuk, G. Karapostoli, T. Lenzi, J. Luetic, T. Maerschalk, A. Marinov, A. Randle-conde, T. Seva, C. Vander Velde, P. Vanlaer, D. Vannerom, R. Yonamine, F. Zenoni, F. Zhang ${ }^{2}$

\section{Ghent University, Ghent, Belgium}

A. Cimmino, T. Cornelis, D. Dobur, A. Fagot, M. Gul, I. Khvastunov, D. Poyraz, C. Roskas, S. Salva, M. Tytgat, W. Verbeke, N. Zaganidis

\section{Université Catholique de Louvain, Louvain-la-Neuve, Belgium}

H. Bakhshiansohi, O. Bondu, S. Brochet, G. Bruno, C. Caputo, A. Caudron, S. De Visscher, C. Delaere, M. Delcourt, B. Francois, A. Giammanco, A. Jafari, M. Komm, G. Krintiras, V. Lemaitre, A. Magitteri, A. Mertens, M. Musich, K. Piotrzkowski, L. Quertenmont, M. Vidal Marono, S. Wertz

\section{Université de Mons, Mons, Belgium}

N. Beliy

\section{Centro Brasileiro de Pesquisas Fisicas, Rio de Janeiro, Brazil}

W.L. Aldá Júnior, F.L. Alves, G.A. Alves, L. Brito, M. Correa Martins Junior, C. Hensel, A. Moraes, M.E. Pol, P. Rebello Teles 
Universidade do Estado do Rio de Janeiro, Rio de Janeiro, Brazil

E. Belchior Batista Das Chagas, W. Carvalho, J. Chinellato ${ }^{3}$, A. Custódio, E.M. Da Costa, G.G. Da Silveira ${ }^{4}$, D. De Jesus Damiao, S. Fonseca De Souza, L.M. Huertas Guativa, H. Malbouisson, M. Melo De Almeida, C. Mora Herrera, L. Mundim, H. Nogima, A. Santoro, A. Sznajder, E.J. Tonelli Manganote ${ }^{3}$, F. Torres Da Silva De Araujo, A. Vilela Pereira

Universidade Estadual Paulista $^{a}$, Universidade Federal do ABC ${ }^{b}$, São Paulo, Brazil

S. Ahuja ${ }^{a}$, C.A. Bernardes ${ }^{a}$, T.R. Fernandez Perez Tomei ${ }^{a}$, E.M. Gregores ${ }^{b}$, P.G. Mercadante ${ }^{b}$, S.F. Novaes ${ }^{a}$, Sandra S. Padula ${ }^{a}$, D. Romero $\operatorname{Abad}^{b}$, J.C. Ruiz $\operatorname{Vargas}^{a}$

Institute for Nuclear Research and Nuclear Energym Bulgarian Academy of Sciences, Sofia, Bulgaria

A. Aleksandrov, R. Hadjiiska, P. Iaydjiev, M. Misheva, M. Rodozov, M. Shopova, S. Stoykova, G. Sultanov

University of Sofia, Sofia, Bulgaria

A. Dimitrov, I. Glushkov, L. Litov, B. Pavlov, P. Petkov

Beihang University, Beijing, China

W. Fang ${ }^{5}$, X. Gao ${ }^{5}$

Institute of High Energy Physics, Beijing, China

M. Ahmad, J.G. Bian, G.M. Chen, H.S. Chen, M. Chen, Y. Chen, C.H. Jiang, D. Leggat, H. Liao, Z. Liu, F. Romeo, S.M. Shaheen, A. Spiezia, J. Tao, C. Wang, Z. Wang, E. Yazgan, H. Zhang, S. Zhang, J. Zhao

State Key Laboratory of Nuclear Physics and Technology, Peking University, Beijing, China

Y. Ban, G. Chen, Q. Li, S. Liu, Y. Mao, S.J. Qian, D. Wang, Z. Xu

Universidad de Los Andes, Bogota, Colombia

C. Avila, A. Cabrera, L.F. Chaparro Sierra, C. Florez, C.F. González Hernández, J.D. Ruiz Alvarez

University of Split, Faculty of Electrical Engineering, Mechanical Engineering and Naval Architecture, Split, Croatia

B. Courbon, N. Godinovic, D. Lelas, I. Puljak, P.M. Ribeiro Cipriano, T. Sculac

University of Split, Faculty of Science, Split, Croatia

Z. Antunovic, M. Kovac

Institute Rudjer Boskovic, Zagreb, Croatia

V. Brigljevic, D. Ferencek, K. Kadija, B. Mesic, A. Starodumov ${ }^{6}$, T. Susa

University of Cyprus, Nicosia, Cyprus

M.W. Ather, A. Attikis, G. Mavromanolakis, J. Mousa, C. Nicolaou, F. Ptochos, P.A. Razis, H. Rykaczewski 
Charles University, Prague, Czech Republic

M. Finger ${ }^{7}$, M. Finger Jr. ${ }^{7}$

Universidad San Francisco de Quito, Quito, Ecuador

E. Carrera Jarrin

Academy of Scientific Research and Technology of the Arab Republic of Egypt, Egyptian Network of High Energy Physics, Cairo, Egypt

A. Ellithi Kamel ${ }^{8}$, S. Khalil ${ }^{9}$, A. Mohamed ${ }^{9}$

National Institute of Chemical Physics and Biophysics, Tallinn, Estonia

R.K. Dewanjee, M. Kadastik, L. Perrini, M. Raidal, A. Tiko, C. Veelken

Department of Physics, University of Helsinki, Helsinki, Finland

P. Eerola, J. Pekkanen, M. Voutilainen

Helsinki Institute of Physics, Helsinki, Finland

J. Härkönen, T. Järvinen, V. Karimäki, R. Kinnunen, T. Lampén, K. Lassila-Perini,

S. Lehti, T. Lindén, P. Luukka, E. Tuominen, J. Tuominiemi, E. Tuovinen

Lappeenranta University of Technology, Lappeenranta, Finland

J. Talvitie, T. Tuuva

IRFU, CEA, Université Paris-Saclay, Gif-sur-Yvette, France

M. Besancon, F. Couderc, M. Dejardin, D. Denegri, J.L. Faure, F. Ferri, S. Ganjour, S. Ghosh, A. Givernaud, P. Gras, G. Hamel de Monchenault, P. Jarry, I. Kucher, E. Locci, M. Machet, J. Malcles, G. Negro, J. Rander, A. Rosowsky, M.Ö. Sahin, M. Titov

Laboratoire Leprince-Ringuet, Ecole polytechnique, CNRS/IN2P3, Université Paris-Saclay, Palaiseau, France

A. Abdulsalam, C. Amendola, I. Antropov, S. Baffioni, F. Beaudette, P. Busson,

L. Cadamuro, C. Charlot, R. Granier de Cassagnac, M. Jo, S. Lisniak, A. Lobanov,

J. Martin Blanco, M. Nguyen, C. Ochando, G. Ortona, P. Paganini, P. Pigard, R. Salerno, J.B. Sauvan, Y. Sirois, A.G. Stahl Leiton, T. Strebler, Y. Yilmaz, A. Zabi, A. Zghiche

Université de Strasbourg, CNRS, IPHC UMR 7178, F-67000 Strasbourg, France

J.-L. Agram ${ }^{10}$, J. Andrea, D. Bloch, J.-M. Brom, M. Buttignol, E.C. Chabert, N. Chanon, C. Collard, E. Conte ${ }^{10}$, X. Coubez, J.-C. Fontaine ${ }^{10}$, D. Gelé, U. Goerlach, M. Jansová, A.-C. Le Bihan, N. Tonon, P. Van Hove

Centre de Calcul de l'Institut National de Physique Nucleaire et de Physique des Particules, CNRS/IN2P3, Villeurbanne, France

S. Gadrat

Université de Lyon, Université Claude Bernard Lyon 1, CNRS-IN2P3, Institut de Physique Nucléaire de Lyon, Villeurbanne, France

S. Beauceron, C. Bernet, G. Boudoul, R. Chierici, D. Contardo, P. Depasse, H. El Mamouni, J. Fay, L. Finco, S. Gascon, M. Gouzevitch, G. Grenier, B. Ille, F. Lagarde, I.B. Laktineh, 
M. Lethuillier, L. Mirabito, A.L. Pequegnot, S. Perries, A. Popov ${ }^{11}$, V. Sordini, M. Vander Donckt, S. Viret

\section{Georgian Technical University, Tbilisi, Georgia}

A. Khvedelidze ${ }^{7}$

Tbilisi State University, Tbilisi, Georgia

Z. Tsamalaidze ${ }^{7}$

RWTH Aachen University, I. Physikalisches Institut, Aachen, Germany

C. Autermann, L. Feld, M.K. Kiesel, K. Klein, M. Lipinski, M. Preuten, C. Schomakers, J. Schulz, T. Verlage, V. Zhukov ${ }^{11}$

RWTH Aachen University, III. Physikalisches Institut A, Aachen, Germany A. Albert, E. Dietz-Laursonn, D. Duchardt, M. Endres, M. Erdmann, S. Erdweg, T. Esch, R. Fischer, A. Güth, M. Hamer, T. Hebbeker, C. Heidemann, K. Hoepfner, S. Knutzen, M. Merschmeyer, A. Meyer, P. Millet, S. Mukherjee, T. Pook, M. Radziej, H. Reithler, M. Rieger, F. Scheuch, D. Teyssier, S. Thüer

RWTH Aachen University, III. Physikalisches Institut B, Aachen, Germany

G. Flügge, B. Kargoll, T. Kress, A. Künsken, J. Lingemann, T. Müller, A. Nehrkorn, A. Nowack, C. Pistone, O. Pooth, A. Stahl ${ }^{12}$

\section{Deutsches Elektronen-Synchrotron, Hamburg, Germany}

M. Aldaya Martin, T. Arndt, C. Asawatangtrakuldee, K. Beernaert, O. Behnke, U. Behrens, A. Bermúdez Martínez, A.A. Bin Anuar, K. Borras ${ }^{13}$, V. Botta, A. Campbell, P. Connor, C. Contreras-Campana, F. Costanza, C. Diez Pardos, G. Eckerlin, D. Eckstein, T. Eichhorn, E. Eren, E. Gallo ${ }^{14}$, J. Garay Garcia, A. Geiser, A. Gizhko, J.M. Grados Luyando, A. Grohsjean, P. Gunnellini, M. Guthoff, A. Harb, J. Hauk, M. Hempel ${ }^{15}$, H. Jung, A. Kalogeropoulos, M. Kasemann, J. Keaveney, C. Kleinwort, I. Korol, D. Krücker, W. Lange, A. Lelek, T. Lenz, J. Leonard, K. Lipka, W. Lohmann ${ }^{15}$, R. Mankel, I.-A. Melzer-Pellmann, A.B. Meyer, G. Mittag, J. Mnich, A. Mussgiller, E. Ntomari, D. Pitzl, A. Raspereza, B. Roland, M. Savitskyi, P. Saxena, R. Shevchenko, S. Spannagel, N. Stefaniuk, G.P. Van Onsem, R. Walsh, Y. Wen, K. Wichmann, C. Wissing, O. Zenaiev

\section{University of Hamburg, Hamburg, Germany}

S. Bein, V. Blobel, M. Centis Vignali, T. Dreyer, E. Garutti, D. Gonzalez, J. Haller, A. Hinzmann, M. Hoffmann, A. Karavdina, R. Klanner, R. Kogler, N. Kovalchuk, S. Kurz, T. Lapsien, I. Marchesini, D. Marconi, M. Meyer, M. Niedziela, D. Nowatschin, F. Pantaleo ${ }^{12}$, T. Peiffer, A. Perieanu, C. Scharf, P. Schleper, A. Schmidt, S. Schumann, J. Schwandt, J. Sonneveld, H. Stadie, G. Steinbrück, F.M. Stober, M. Stöver, H. Tholen, D. Troendle, E. Usai, L. Vanelderen, A. Vanhoefer, B. Vormwald

Institut für Experimentelle Kernphysik, Karlsruhe, Germany

M. Akbiyik, C. Barth, S. Baur, E. Butz, R. Caspart, T. Chwalek, F. Colombo, W. De Boer, A. Dierlamm, B. Freund, R. Friese, M. Giffels, D. Haitz, F. Hartmann ${ }^{12}$, S.M. Heindl, 
U. Husemann, F. Kassel ${ }^{12}$, S. Kudella, H. Mildner, M.U. Mozer, Th. Müller, M. Plagge,

G. Quast, K. Rabbertz, M. Schröder, I. Shvetsov, G. Sieber, H.J. Simonis, R. Ulrich, S. Wayand, M. Weber, T. Weiler, S. Williamson, C. Wöhrmann, R. Wolf

Institute of Nuclear and Particle Physics (INPP), NCSR Demokritos, Aghia Paraskevi, Greece

G. Anagnostou, G. Daskalakis, T. Geralis, V.A. Giakoumopoulou, A. Kyriakis, D. Loukas,

I. Topsis-Giotis

National and Kapodistrian University of Athens, Athens, Greece

G. Karathanasis, S. Kesisoglou, A. Panagiotou, N. Saoulidou

National Technical University of Athens, Athens, Greece

K. Kousouris

University of Ioánnina, Ioánnina, Greece

I. Evangelou, C. Foudas, P. Kokkas, S. Mallios, N. Manthos, I. Papadopoulos, E. Paradas, J. Strologas, F.A. Triantis

MTA-ELTE Lendület CMS Particle and Nuclear Physics Group, Eötvös Loránd University, Budapest, Hungary

M. Csanad, N. Filipovic, G. Pasztor, G.I. Veres ${ }^{16}$

Wigner Research Centre for Physics, Budapest, Hungary

G. Bencze, C. Hajdu, D. Horvath ${ }^{17}$, Á. Hunyadi, F. Sikler, V. Veszpremi, A.J. Zsigmond

Institute of Nuclear Research ATOMKI, Debrecen, Hungary

N. Beni, S. Czellar, J. Karancsi ${ }^{18}$, A. Makovec, J. Molnar, Z. Szillasi

Institute of Physics, University of Debrecen, Debrecen, Hungary

M. Bartók ${ }^{16}$, P. Raics, Z.L. Trocsanyi, B. Ujvari

Indian Institute of Science (IISc), Bangalore, India

S. Choudhury, J.R. Komaragiri

National Institute of Science Education and Research, Bhubaneswar, India

S. Bahinipati ${ }^{19}$, S. Bhowmik, P. Mal, K. Mandal, A. Nayak ${ }^{20}$, D.K. Sahoo ${ }^{19}$, N. Sahoo, S.K. Swain

Panjab University, Chandigarh, India

S. Bansal, S.B. Beri, V. Bhatnagar, R. Chawla, N. Dhingra, A.K. Kalsi, A. Kaur, M. Kaur, R. Kumar, P. Kumari, A. Mehta, J.B. Singh, G. Walia

University of Delhi, Delhi, India

Ashok Kumar, Aashaq Shah, A. Bhardwaj, S. Chauhan, B.C. Choudhary, R.B. Garg, S. Keshri, A. Kumar, S. Malhotra, M. Naimuddin, K. Ranjan, R. Sharma

Saha Institute of Nuclear Physics, HBNI, Kolkata, India

R. Bhardwaj, R. Bhattacharya, S. Bhattacharya, U. Bhawandeep, S. Dey, S. Dutt, S. Dutta,

S. Ghosh, N. Majumdar, A. Modak, K. Mondal, S. Mukhopadhyay, S. Nandan, A. Purohit, A. Roy, D. Roy, S. Roy Chowdhury, S. Sarkar, M. Sharan, S. Thakur 
Indian Institute of Technology Madras, Madras, India

P.K. Behera

Bhabha Atomic Research Centre, Mumbai, India

R. Chudasama, D. Dutta, V. Jha, V. Kumar, A.K. Mohanty ${ }^{12}$, P.K. Netrakanti, L.M. Pant, P. Shukla, A. Topkar

Tata Institute of Fundamental Research-A, Mumbai, India

T. Aziz, S. Dugad, B. Mahakud, S. Mitra, G.B. Mohanty, N. Sur, B. Sutar

Tata Institute of Fundamental Research-B, Mumbai, India

S. Banerjee, S. Bhattacharya, S. Chatterjee, P. Das, M. Guchait, Sa. Jain, S. Kumar, M. Maity ${ }^{21}$, G. Majumder, K. Mazumdar, T. Sarkar ${ }^{21}$, N. Wickramage ${ }^{22}$

Indian Institute of Science Education and Research (IISER), Pune, India

S. Chauhan, S. Dube, V. Hegde, A. Kapoor, K. Kothekar, S. Pandey, A. Rane, S. Sharma

Institute for Research in Fundamental Sciences (IPM), Tehran, Iran

S. Chenarani ${ }^{23}$, E. Eskandari Tadavani, S.M. Etesami ${ }^{23}$, M. Khakzad, M. Mohammadi Najafabadi, M. Naseri, S. Paktinat Mehdiabadi ${ }^{24}$, F. Rezaei Hosseinabadi, B. Safarzadeh ${ }^{25}$, M. Zeinali

University College Dublin, Dublin, Ireland

M. Felcini, M. Grunewald

INFN Sezione di Bari ${ }^{a}$, Università di Bari ${ }^{b}$, Politecnico di Bari ${ }^{c}$, Bari, Italy M. Abbrescia ${ }^{a, b}$, C. Calabria ${ }^{a, b}$, A. Colaleo ${ }^{a}$, D. Creanza ${ }^{a, c}$, L. Cristella ${ }^{a, b}$, N. De

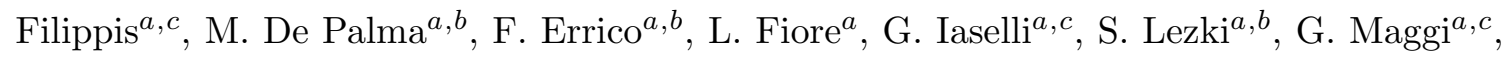
M. Maggi ${ }^{a}$, G. Miniello ${ }^{a, b}$, S. My ${ }^{a, b}$, S. Nuzzo ${ }^{a, b}$, A. Pompili ${ }^{a, b}$, G. Pugliese ${ }^{a, c}$, R. Radogna ${ }^{a}$, A. Ranieri ${ }^{a}$, G. Selvaggi ${ }^{a, b}$, A. Sharma ${ }^{a}$, L. Silvestris ${ }^{a}, 12$, R. $\operatorname{Venditti}^{a}$, P. Verwilligen ${ }^{a}$

INFN Sezione di Bologna ${ }^{a}$, Università di Bologna ${ }^{b}$, Bologna, Italy

G. Abbiendi ${ }^{a}$, C. Battilana ${ }^{a, b}$, D. Bonacorsi ${ }^{a}, b$, S. Braibant-Giacomelli ${ }^{a, b}$, R. Campanini $^{a, b}$, P. Capiluppi ${ }^{a, b}$, A. Castro ${ }^{a, b}$, F.R. Cavallo ${ }^{a}$, S.S. Chhibra ${ }^{a}$, G. Codispoti ${ }^{a, b}$, M. Cuffiani ${ }^{a, b}$, G.M. Dallavalle ${ }^{a}$, F. Fabbri ${ }^{a}$, A. Fanfani ${ }^{a}, b$, D. Fasanella ${ }^{a, b}$, P. Giacomelli ${ }^{a}$, C. Grandi ${ }^{a}$, L. Guiducci ${ }^{a}, b$, S. Marcellini ${ }^{a}$, G. Masetti ${ }^{a}$, A. Montanari ${ }^{a}$, F.L. Navarria ${ }^{a, b}$, A. Perrotta ${ }^{a}$, A.M. Rossi ${ }^{a, b}$, T. Rovelli ${ }^{a, b}$, G.P. Siroli ${ }^{a, b}$, N. Tosi ${ }^{a}$

INFN Sezione di Catania ${ }^{a}$, Università di Catania ${ }^{b}$, Catania, Italy

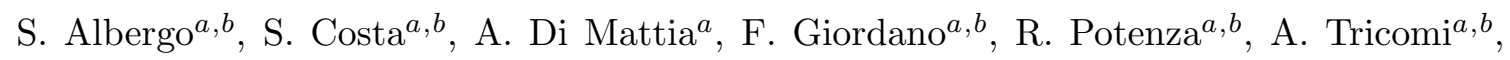
C. Tuve ${ }^{a, b}$

INFN Sezione di Firenze ${ }^{a}$, Università di Firenze ${ }^{b}$, Firenze, Italy

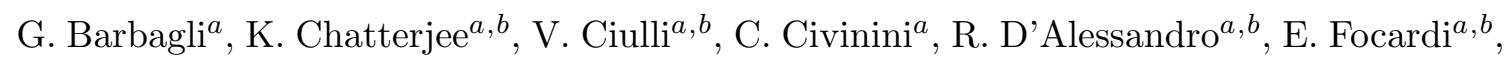

P. Lenzi ${ }^{a, b}$, M. Meschini ${ }^{a}$, S. Paoletti ${ }^{a}$, L. Russo ${ }^{a, 26}$, G. Sguazzoni ${ }^{a}$, D. Strom $^{a}$,

L. Viliani ${ }^{a, b, 12}$ 
INFN Laboratori Nazionali di Frascati, Frascati, Italy

L. Benussi, S. Bianco, F. Fabbri, D. Piccolo, F. Primavera ${ }^{12}$

INFN Sezione di Genova ${ }^{a}$, Università di Genova ${ }^{b}$, Genova, Italy

V. Calvelli ${ }^{a, b}$, F. Ferro $^{a}$, E. Robutti ${ }^{a}$, S. Tosi ${ }^{a, b}$

INFN Sezione di Milano-Bicocca ${ }^{a}$, Università di Milano-Bicocca ${ }^{b}$, Milano, Italy

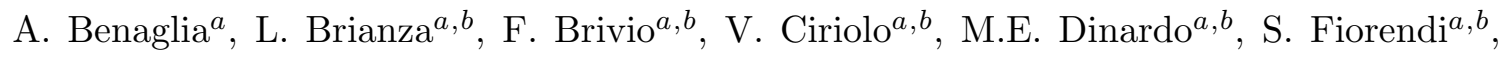

S. Gennai ${ }^{a}$, A. Ghezzi ${ }^{a, b}$, P. Govoni ${ }^{a, b}$, M. Malberti ${ }^{a, b}$, S. Malvezzi ${ }^{a}$, R.A. Manzoni ${ }^{a, b}$,

D. Menasce ${ }^{a}$, L. Moroni ${ }^{a}$, M. Paganoni ${ }^{a, b}$, K. Pauwels ${ }^{a, b}$, D. Pedrini ${ }^{a}$, S. Pigazzini ${ }^{a}, b, 27$,

S. Ragazzi ${ }^{a, b}$, N. Redaelli ${ }^{a}$, T. Tabarelli de Fatis ${ }^{a, b}$

INFN Sezione di Napoli ${ }^{a}$, Università di Napoli 'Federico II' ${ }^{b}$, Napoli, Italy, Università della Basilicata ${ }^{c}$, Potenza, Italy, Università G. Marconi ${ }^{d}$, Roma, Italy

S. Buontempo ${ }^{a}$, N. Cavallo ${ }^{a, c}$, S. Di Guida ${ }^{a, d, 12}$, F. Fabozzi ${ }^{a, c}$, F. Fienga ${ }^{a, b}$,

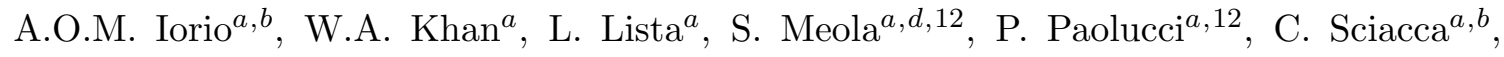
F. Thyssen ${ }^{a}$

INFN Sezione di Padova ${ }^{a}$, Università di Padova ${ }^{b}$, Padova, Italy, Università di Trento ${ }^{c}$, Trento, Italy

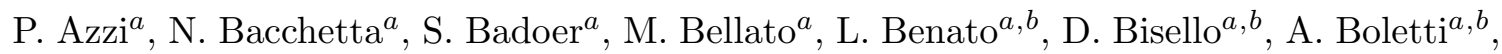

A. Carvalho Antunes De Oliveira ${ }^{a, b}$, P. Checchia ${ }^{a}$, M. Dall'Osso $^{a, b}$, P. De Castro Manzano ${ }^{a}$,

T. Dorigo ${ }^{a}$, U. Gasparini ${ }^{a, b}$, A. Gozzelino ${ }^{a}$, S. Lacaprara ${ }^{a}$, P. Lujan, M. Margoni ${ }^{a}, b$, A.T. Meneguzzo ${ }^{a, b}$, N. Pozzobon ${ }^{a, b}$, P. Ronchese ${ }^{a, b}$, R. $\operatorname{Rossin}^{a, b}$, F. Simonetto ${ }^{a, b}$, E. Torassa ${ }^{a}$, M. Zanetti ${ }^{a, b}$, P. Zotto ${ }^{a, b}$, G. Zumerle ${ }^{a, b}$

INFN Sezione di Pavia ${ }^{a}$, Università di Pavia ${ }^{b}$, Pavia, Italy

A. Braghieri ${ }^{a}$, A. Magnani ${ }^{a}$, P. Montagna ${ }^{a, b}$, S.P. Ratti ${ }^{a, b}$, V. $\operatorname{Re}^{a}$, M. Ressegotti $^{a, b}$, C. Riccardi ${ }^{a, b}$, P. Salvini ${ }^{a}$, I. Vai ${ }^{a}, b$, P. Vitulo ${ }^{a, b}$

INFN Sezione di Perugia ${ }^{a}$, Università di Perugia ${ }^{b}$, Perugia, Italy

L. Alunni Solestizi ${ }^{a, b}$, M. Biasini ${ }^{a, b}$, G.M. Bilei ${ }^{a}$, C. Cecchi $^{a, b}$, D. Ciangottini ${ }^{a, b}$, L. Fanò $^{a, b}$,

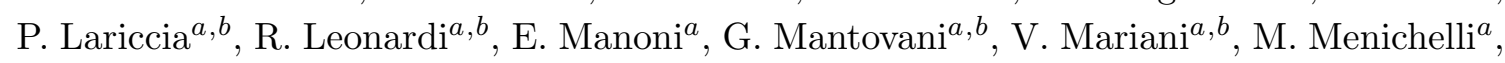
A. $\operatorname{Rossi}^{a, b}$, A. Santocchia ${ }^{a, b}$, D. Spiga ${ }^{a}$

INFN Sezione di Pisa ${ }^{a}$, Università di Pisa ${ }^{b}$, Scuola Normale Superiore di Pisa ${ }^{c}$, Pisa, Italy

K. Androsov ${ }^{a}$, P. Azzurri ${ }^{a, 12}$, G. Bagliesi ${ }^{a}$, T. Boccali ${ }^{a}$, L. Borrello, R. Castaldi ${ }^{a}$, M.A. Ciocci ${ }^{a, b}$, R. Dell'Orso ${ }^{a}$, G. Fedi ${ }^{a}$, L. Giannini ${ }^{a, c}$, A. Giassi ${ }^{a}$, M.T. Grippo $^{a, 26}$, F. Ligabue ${ }^{a, c}$, T. Lomtadze ${ }^{a}$, E. Manca ${ }^{a, c}$, G. Mandorli ${ }^{a, c}$, L. Martini ${ }^{a, b}$, A. Messineo ${ }^{a, b}$, F. Palla ${ }^{a}$, A. Rizzi ${ }^{a, b}$, A. Savoy-Navarro ${ }^{a, 28}$, P. Spagnolo ${ }^{a}$, R. Tenchini ${ }^{a}$, G. Tonelli ${ }^{a, b}$, A. Venturi ${ }^{a}$, P.G. Verdini ${ }^{a}$

INFN Sezione di Roma ${ }^{a}$, Sapienza Università di Roma ${ }^{b}$, Rome, Italy

L. Barone ${ }^{a, b}$, F. Cavallari ${ }^{a}$, M. Cipriani ${ }^{a, b}$, N. Daci ${ }^{a}$, D. Del Re Re $^{a, b, 12}$, E. Di $\operatorname{Marco}^{a, b}$, M. Diemoz ${ }^{a}$, S. Gelli ${ }^{a, b}$, E. Longo ${ }^{a, b}$, F. Margaroli ${ }^{a, b}$, B. Marzocchi ${ }^{a, b}$, 


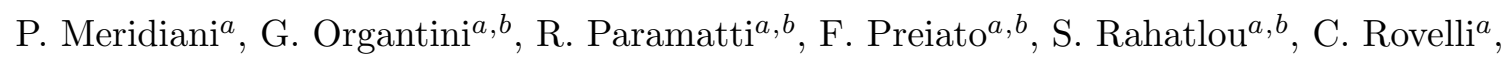
F. Santanastasio ${ }^{a, b}$

INFN Sezione di Torino ${ }^{a}$, Università di Torino ${ }^{b}$, Torino, Italy, Università del Piemonte Orientale ${ }^{c}$, Novara, Italy

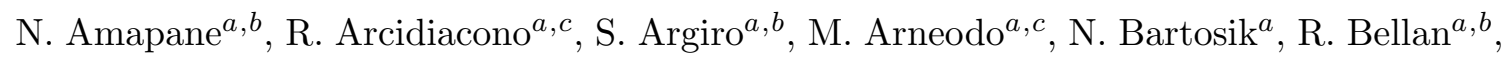

C. Biino ${ }^{a}$, N. Cartiglia ${ }^{a}$, F. Cenna ${ }^{a, b}$, M. Costa ${ }^{a, b}$, R. Covarelli ${ }^{a, b}$, A. Degano ${ }^{a, b}$,

N. $\operatorname{Demaria}^{a}$, B. Kiani ${ }^{a, b}$, C. Mariotti $^{a}$, S. Maselli ${ }^{a}$, E. Migliore ${ }^{a, b}$, V. Monaco ${ }^{a, b}$,

E. Monteil $^{a, b}$, M. Monteno $^{a}$, M.M. Obertino $^{a, b}$, L. Pacher $^{a}, b$, N. Pastrone $^{a}$, M. Pelliccioni $^{a}$,

G.L. Pinna Angioni ${ }^{a, b}$, F. Ravera ${ }^{a, b}$, A. Romero ${ }^{a, b}$, M. Ruspa ${ }^{a, c}$, R. Sacchi ${ }^{a, b}$, K. Shchelina ${ }^{a, b}$, V. Sola ${ }^{a}$, A. Solano ${ }^{a, b}$, A. Staiano ${ }^{a}$, P. Traczyk $^{a, b}$

INFN Sezione di Trieste ${ }^{a}$, Università di Trieste ${ }^{b}$, Trieste, Italy

S. Belforte ${ }^{a}$, M. Casarsa ${ }^{a}$, F. Cossutti ${ }^{a}$, G. Della Ricca ${ }^{a, b}$, A. Zanetti $^{a}$

Kyungpook National University, Daegu, Korea

D.H. Kim, G.N. Kim, M.S. Kim, J. Lee, S. Lee, S.W. Lee, C.S. Moon, Y.D. Oh, S. Sekmen, D.C. Son, Y.C. Yang

Chonbuk National University, Jeonju, Korea

A. Lee

Chonnam National University, Institute for Universe and Elementary Particles, Kwangju, Korea

H. Kim, D.H. Moon, G. Oh

Hanyang University, Seoul, Korea

J.A. Brochero Cifuentes, J. Goh, T.J. Kim

Korea University, Seoul, Korea

S. Cho, S. Choi, Y. Go, D. Gyun, S. Ha, B. Hong, Y. Jo, Y. Kim, K. Lee, K.S. Lee, S. Lee, J. Lim, S.K. Park, Y. Roh

Seoul National University, Seoul, Korea

J. Almond, J. Kim, J.S. Kim, H. Lee, K. Lee, K. Nam, S.B. Oh, B.C. Radburn-Smith, S.h. Seo, U.K. Yang, H.D. Yoo, G.B. Yu

University of Seoul, Seoul, Korea

M. Choi, H. Kim, J.H. Kim, J.S.H. Lee, I.C. Park

Sungkyunkwan University, Suwon, Korea

Y. Choi, C. Hwang, J. Lee, I. Yu

Vilnius University, Vilnius, Lithuania

V. Dudenas, A. Juodagalvis, J. Vaitkus

National Centre for Particle Physics, Universiti Malaya, Kuala Lumpur, Malaysia

I. Ahmed, Z.A. Ibrahim, M.A.B. Md Ali ${ }^{29}$, F. Mohamad Idris ${ }^{30}$, W.A.T. Wan Abdullah, M.N. Yusli, Z. Zolkapli 
Centro de Investigacion y de Estudios Avanzados del IPN, Mexico City, Mexico Reyes-Almanza, R, Ramirez-Sanchez, G., Duran-Osuna, M. C., H. Castilla-Valdez, E. De La Cruz-Burelo, I. Heredia-De La Cruz ${ }^{31}$, Rabadan-Trejo, R. I., R. Lopez-Fernandez, J. Mejia Guisao, A. Sanchez-Hernandez

Universidad Iberoamericana, Mexico City, Mexico

S. Carrillo Moreno, C. Oropeza Barrera, F. Vazquez Valencia

Benemerita Universidad Autonoma de Puebla, Puebla, Mexico

I. Pedraza, H.A. Salazar Ibarguen, C. Uribe Estrada

Universidad Autónoma de San Luis Potosí, San Luis Potosí, Mexico

A. Morelos Pineda

University of Auckland, Auckland, New Zealand

D. Krofcheck

University of Canterbury, Christchurch, New Zealand

P.H. Butler

National Centre for Physics, Quaid-I-Azam University, Islamabad, Pakistan

A. Ahmad, M. Ahmad, Q. Hassan, H.R. Hoorani, A. Saddique, M.A. Shah, M. Shoaib, M. Waqas

National Centre for Nuclear Research, Swierk, Poland

H. Bialkowska, M. Bluj, B. Boimska, T. Frueboes, M. Górski, M. Kazana, K. Nawrocki, M. Szleper, P. Zalewski

Institute of Experimental Physics, Faculty of Physics, University of Warsaw, Warsaw, Poland

K. Bunkowski, A. Byszuk ${ }^{32}$, K. Doroba, A. Kalinowski, M. Konecki, J. Krolikowski, M. Misiura, M. Olszewski, A. Pyskir, M. Walczak

Laboratório de Instrumentação e Física Experimental de Partículas, Lisboa, Portugal

P. Bargassa, C. Beirão Da Cruz E Silva, A. Di Francesco, P. Faccioli, B. Galinhas, M. Gallinaro, J. Hollar, N. Leonardo, L. Lloret Iglesias, M.V. Nemallapudi, J. Seixas, G. Strong, O. Toldaiev, D. Vadruccio, J. Varela

Joint Institute for Nuclear Research, Dubna, Russia

S. Afanasiev, P. Bunin, M. Gavrilenko, I. Golutvin, I. Gorbunov, A. Kamenev, V. Karjavin, A. Lanev, A. Malakhov, V. Matveev ${ }^{33,34}$, V. Palichik, V. Perelygin, S. Shmatov, S. Shulha, N. Skatchkov, V. Smirnov, N. Voytishin, A. Zarubin

Petersburg Nuclear Physics Institute, Gatchina (St. Petersburg), Russia Y. Ivanov, V. Kim ${ }^{35}$, E. Kuznetsova ${ }^{36}$, P. Levchenko, V. Murzin, V. Oreshkin, I. Smirnov, V. Sulimov, L. Uvarov, S. Vavilov, A. Vorobyev 
Institute for Nuclear Research, Moscow, Russia

Yu. Andreev, A. Dermenev, S. Gninenko, N. Golubev, A. Karneyeu, M. Kirsanov, N. Krasnikov, A. Pashenkov, D. Tlisov, A. Toropin

Institute for Theoretical and Experimental Physics, Moscow, Russia

V. Epshteyn, V. Gavrilov, N. Lychkovskaya, V. Popov, I. Pozdnyakov, G. Safronov, A. Spiridonov, A. Stepennov, M. Toms, E. Vlasov, A. Zhokin

Moscow Institute of Physics and Technology, Moscow, Russia

T. Aushev, A. Bylinkin ${ }^{34}$

National Research Nuclear University 'Moscow Engineering Physics Institute' (MEPhI), Moscow, Russia

M. Chadeeva ${ }^{37}$, P. Parygin, D. Philippov, S. Polikarpov, E. Popova, V. Rusinov

P.N. Lebedev Physical Institute, Moscow, Russia

V. Andreev, M. Azarkin ${ }^{34}$, I. Dremin ${ }^{34}$, M. Kirakosyan ${ }^{34}$, A. Terkulov

Skobeltsyn Institute of Nuclear Physics, Lomonosov Moscow State University, Moscow, Russia

A. Baskakov, A. Belyaev, E. Boos, M. Dubinin ${ }^{38}$, L. Dudko, A. Ershov, A. Gribushin, V. Klyukhin, O. Kodolova, I. Lokhtin, I. Miagkov, S. Obraztsov, S. Petrushanko, V. Savrin, A. Snigirev

Novosibirsk State University (NSU), Novosibirsk, Russia

V. Blinov ${ }^{39}$, Y.Skovpen ${ }^{39}$, D. Shtol ${ }^{39}$

State Research Center of Russian Federation, Institute for High Energy Physics, Protvino, Russia

I. Azhgirey, I. Bayshev, S. Bitioukov, D. Elumakhov, V. Kachanov, A. Kalinin, D. Konstantinov, V. Petrov, R. Ryutin, A. Sobol, S. Troshin, N. Tyurin, A. Uzunian, A. Volkov

University of Belgrade, Faculty of Physics and Vinca Institute of Nuclear Sciences, Belgrade, Serbia

P. Adzic ${ }^{40}$, P. Cirkovic, D. Devetak, M. Dordevic, J. Milosevic, V. Rekovic

Centro de Investigaciones Energéticas Medioambientales y Tecnológicas (CIEMAT), Madrid, Spain

J. Alcaraz Maestre, M. Barrio Luna, M. Cerrada, N. Colino, B. De La Cruz, A. Delgado Peris, A. Escalante Del Valle, C. Fernandez Bedoya, J.P. Fernández Ramos, J. Flix, M.C. Fouz, P. Garcia-Abia, O. Gonzalez Lopez, S. Goy Lopez, J.M. Hernandez, M.I. Josa, D. Moran, A. Pérez-Calero Yzquierdo, J. Puerta Pelayo, A. Quintario Olmeda, I. Redondo, L. Romero, M.S. Soares, A. Álvarez Fernández

Universidad Autónoma de Madrid, Madrid, Spain

C. Albajar, J.F. de Trocóniz, M. Missiroli 


\section{Universidad de Oviedo, Oviedo, Spain}

J. Cuevas, C. Erice, J. Fernandez Menendez, I. Gonzalez Caballero, J.R. González Fernández, E. Palencia Cortezon, S. Sanchez Cruz, P. Vischia, J.M. Vizan Garcia

Instituto de Física de Cantabria (IFCA), CSIC-Universidad de Cantabria, Santander, Spain

I.J. Cabrillo, A. Calderon, B. Chazin Quero, E. Curras, J. Duarte Campderros, M. Fernandez, J. Garcia-Ferrero, G. Gomez, A. Lopez Virto, J. Marco, C. Martinez Rivero, P. Martinez Ruiz del Arbol, F. Matorras, J. Piedra Gomez, T. Rodrigo, A. Ruiz-Jimeno, L. Scodellaro, N. Trevisani, I. Vila, R. Vilar Cortabitarte

\section{CERN, European Organization for Nuclear Research, Geneva, Switzerland}

D. Abbaneo, E. Auffray, P. Baillon, A.H. Ball, D. Barney, M. Bianco, P. Bloch, A. Bocci,

C. Botta, T. Camporesi, R. Castello, M. Cepeda, G. Cerminara, E. Chapon, Y. Chen, D. d'Enterria, A. Dabrowski, V. Daponte, A. David, M. De Gruttola, A. De Roeck, M. Dobson, B. Dorney, T. du Pree, M. Dünser, N. Dupont, A. Elliott-Peisert, P. Everaerts, F. Fallavollita, G. Franzoni, J. Fulcher, W. Funk, D. Gigi, A. Gilbert, K. Gill, F. Glege, D. Gulhan, P. Harris, J. Hegeman, V. Innocente, P. Janot, O. Karacheban ${ }^{15}$, J. Kieseler, H. Kirschenmann, V. Knünz, A. Kornmayer ${ }^{12}$, M.J. Kortelainen, M. Krammer ${ }^{1}$, C. Lange, P. Lecoq, C. Lourenço, M.T. Lucchini, L. Malgeri, M. Mannelli, A. Martelli, F. Meijers, J.A. Merlin, S. Mersi, E. Meschi, P. Milenovic ${ }^{41}$, F. Moortgat, M. Mulders, H. Neugebauer, J. Ngadiuba, S. Orfanelli, L. Orsini, L. Pape, E. Perez, M. Peruzzi, A. Petrilli, G. Petrucciani, A. Pfeiffer, M. Pierini, A. Racz, T. Reis, G. Rolandi ${ }^{42}$, M. Rovere, H. Sakulin, C. Schäfer, C. Schwick, M. Seidel, M. Selvaggi, A. Sharma, P. Silva, P. Sphicas ${ }^{43}$, A. Stakia, J. Steggemann, M. Stoye, M. Tosi, D. Treille, A. Triossi, A. Tsirou, V. Veckalns ${ }^{44}$, M. Verweij, W.D. Zeuner

\section{Paul Scherrer Institut, Villigen, Switzerland}

W. Bertl ${ }^{\dagger}$, L. Caminada ${ }^{45}$, K. Deiters, W. Erdmann, R. Horisberger, Q. Ingram, H.C. Kaestli, D. Kotlinski, U. Langenegger, T. Rohe, S.A. Wiederkehr

ETH Zurich - Institute for Particle Physics and Astrophysics (IPA), Zurich, Switzerland

L. Bäni, P. Berger, L. Bianchini, B. Casal, G. Dissertori, M. Dittmar, M. Donegà, C. Grab, C. Heidegger, D. Hits, J. Hoss, G. Kasieczka, T. Klijnsma, W. Lustermann, B. Mangano, M. Marionneau, M.T. Meinhard, D. Meister, F. Micheli, P. Musella, F. Nessi-Tedaldi, F. Pandolfi, J. Pata, F. Pauss, G. Perrin, L. Perrozzi, M. Quittnat, M. Reichmann, M. Schönenberger, L. Shchutska, V.R. Tavolaro, K. Theofilatos, M.L. Vesterbacka Olsson, R. Wallny, D.H. Zhu

\section{Universität Zürich, Zurich, Switzerland}

T.K. Aarrestad, C. Amsler ${ }^{46}$, M.F. Canelli, A. De Cosa, R. Del Burgo, S. Donato, C. Galloni, T. Hreus, B. Kilminster, D. Pinna, G. Rauco, P. Robmann, D. Salerno, C. Seitz, Y. Takahashi, A. Zucchetta 
National Central University, Chung-Li, Taiwan

V. Candelise, T.H. Doan, Sh. Jain, R. Khurana, C.M. Kuo, W. Lin, A. Pozdnyakov, S.S. Yu

National Taiwan University (NTU), Taipei, Taiwan

Arun Kumar, P. Chang, Y. Chao, K.F. Chen, P.H. Chen, F. Fiori, W.-S. Hou, Y. Hsiung, Y.F. Liu, R.-S. Lu, E. Paganis, A. Psallidas, A. Steen, J.f. Tsai

Chulalongkorn University, Faculty of Science, Department of Physics, Bangkok, Thailand

B. Asavapibhop, K. Kovitanggoon, G. Singh, N. Srimanobhas

Çukurova University, Physics Department, Science and Art Faculty, Adana, Turkey

F. Boran, S. Cerci ${ }^{47}$, S. Damarseckin, Z.S. Demiroglu, C. Dozen, I. Dumanoglu, S. Girgis,

G. Gokbulut, Y. Guler, I. Hos $^{48}$, E.E. Kangal ${ }^{49}$, O. Kara, A. Kayis Topaksu, U. Kiminsu,

M. Oglakci, G. Onengut ${ }^{50}$, K. Ozdemir ${ }^{51}$, D. Sunar Cerci $^{47}$, B. Tali ${ }^{47}$, S. Turkcapar, I.S. Zorbakir, C. Zorbilmez

Middle East Technical University, Physics Department, Ankara, Turkey

B. Bilin, G. Karapinar ${ }^{52}$, K. Ocalan ${ }^{53}$, M. Yalvac, M. Zeyrek

Bogazici University, Istanbul, Turkey

E. Gülmez, M. Kaya ${ }^{54}$, O. Kaya ${ }^{55}$, S. Tekten, E.A. Yetkin ${ }^{56}$

Istanbul Technical University, Istanbul, Turkey

M.N. Agaras, S. Atay, A. Cakir, K. Cankocak

Institute for Scintillation Materials of National Academy of Science of Ukraine, Kharkov, Ukraine

B. Grynyov

National Scientific Center, Kharkov Institute of Physics and Technology, Kharkov, Ukraine

L. Levchuk

University of Bristol, Bristol, United Kingdom

R. Aggleton, F. Ball, L. Beck, J.J. Brooke, D. Burns, E. Clement, D. Cussans, O. Davignon,

H. Flacher, J. Goldstein, M. Grimes, G.P. Heath, H.F. Heath, J. Jacob, L. Kreczko,

C. Lucas, D.M. Newbold ${ }^{57}$, S. Paramesvaran, A. Poll, T. Sakuma, S. Seif El Nasr-storey,

D. Smith, V.J. Smith

Rutherford Appleton Laboratory, Didcot, United Kingdom

K.W. Bell, A. Belyaev ${ }^{58}$, C. Brew, R.M. Brown, L. Calligaris, D. Cieri, D.J.A. Cockerill, J.A. Coughlan, K. Harder, S. Harper, E. Olaiya, D. Petyt, C.H. Shepherd-Themistocleous, A. Thea, I.R. Tomalin, T. Williams

Imperial College, London, United Kingdom

G. Auzinger, R. Bainbridge, J. Borg, S. Breeze, O. Buchmuller, A. Bundock, S. Casasso, M. Citron, D. Colling, L. Corpe, P. Dauncey, G. Davies, A. De Wit, M. Della Negra, R. Di 
Maria, A. Elwood, Y. Haddad, G. Hall, G. Iles, T. James, R. Lane, C. Laner, L. Lyons, A.-M. Magnan, S. Malik, L. Mastrolorenzo, T. Matsushita, J. Nash, A. Nikitenko ${ }^{6}$, V. Palladino, M. Pesaresi, D.M. Raymond, A. Richards, A. Rose, E. Scott, C. Seez, A. Shtipliyski, S. Summers, A. Tapper, K. Uchida, M. Vazquez Acosta ${ }^{59}$, T. Virdee ${ }^{12}$, N. Wardle, D. Winterbottom, J. Wright, S.C. Zenz

Brunel University, Uxbridge, United Kingdom

J.E. Cole, P.R. Hobson, A. Khan, P. Kyberd, I.D. Reid, P. Symonds, L. Teodorescu, M. Turner

Baylor University, Waco, U.S.A.

A. Borzou, K. Call, J. Dittmann, K. Hatakeyama, H. Liu, N. Pastika, C. Smith

Catholic University of America, Washington DC, U.S.A.

R. Bartek, A. Dominguez

The University of Alabama, Tuscaloosa, U.S.A.

A. Buccilli, S.I. Cooper, C. Henderson, P. Rumerio, C. West

Boston University, Boston, U.S.A.

D. Arcaro, A. Avetisyan, T. Bose, D. Gastler, D. Rankin, C. Richardson, J. Rohlf, L. Sulak, D. Zou

Brown University, Providence, U.S.A.

G. Benelli, D. Cutts, A. Garabedian, J. Hakala, U. Heintz, J.M. Hogan, K.H.M. Kwok, E. Laird, G. Landsberg, Z. Mao, M. Narain, J. Pazzini, S. Piperov, S. Sagir, R. Syarif, D. $\mathrm{Yu}$

University of California, Davis, Davis, U.S.A.

R. Band, C. Brainerd, D. Burns, M. Calderon De La Barca Sanchez, M. Chertok, J. Conway, R. Conway, P.T. Cox, R. Erbacher, C. Flores, G. Funk, M. Gardner, W. Ko, R. Lander, C. Mclean, M. Mulhearn, D. Pellett, J. Pilot, S. Shalhout, M. Shi, J. Smith, D. Stolp, K. Tos, M. Tripathi, Z. Wang

University of California, Los Angeles, U.S.A.

M. Bachtis, C. Bravo, R. Cousins, A. Dasgupta, A. Florent, J. Hauser, M. Ignatenko, N. Mccoll, S. Regnard, D. Saltzberg, C. Schnaible, V. Valuev

University of California, Riverside, Riverside, U.S.A.

E. Bouvier, K. Burt, R. Clare, J. Ellison, J.W. Gary, S.M.A. Ghiasi Shirazi, G. Hanson, J. Heilman, P. Jandir, E. Kennedy, F. Lacroix, O.R. Long, M. Olmedo Negrete, M.I. Paneva, A. Shrinivas, W. Si, L. Wang, H. Wei, S. Wimpenny, B. R. Yates

University of California, San Diego, La Jolla, U.S.A.

J.G. Branson, S. Cittolin, M. Derdzinski, R. Gerosa, B. Hashemi, A. Holzner, D. Klein, G. Kole, V. Krutelyov, J. Letts, I. Macneill, M. Masciovecchio, D. Olivito, S. Padhi, M. Pieri, M. Sani, V. Sharma, S. Simon, M. Tadel, A. Vartak, S. Wasserbaech ${ }^{60}$, J. Wood, F. Würthwein, A. Yagil, G. Zevi Della Porta 
University of California, Santa Barbara - Department of Physics, Santa Barbara, U.S.A.

N. Amin, R. Bhandari, J. Bradmiller-Feld, C. Campagnari, A. Dishaw, V. Dutta, M. Franco Sevilla, C. George, F. Golf, L. Gouskos, J. Gran, R. Heller, J. Incandela, S.D. Mullin, A. Ovcharova, H. Qu, J. Richman, D. Stuart, I. Suarez, J. Yoo

California Institute of Technology, Pasadena, U.S.A.

D. Anderson, J. Bendavid, A. Bornheim, J.M. Lawhorn, H.B. Newman, T. Nguyen, C. Pena, M. Spiropulu, J.R. Vlimant, S. Xie, Z. Zhang, R.Y. Zhu

Carnegie Mellon University, Pittsburgh, U.S.A.

M.B. Andrews, T. Ferguson, T. Mudholkar, M. Paulini, J. Russ, M. Sun, H. Vogel, I. Vorobiev, M. Weinberg

University of Colorado Boulder, Boulder, U.S.A.

J.P. Cumalat, W.T. Ford, F. Jensen, A. Johnson, M. Krohn, S. Leontsinis, T. Mulholland, K. Stenson, S.R. Wagner

Cornell University, Ithaca, U.S.A.

J. Alexander, J. Chaves, J. Chu, S. Dittmer, K. Mcdermott, N. Mirman, J.R. Patterson, A. Rinkevicius, A. Ryd, L. Skinnari, L. Soffi, S.M. Tan, Z. Tao, J. Thom, J. Tucker, P. Wittich, M. Zientek

\section{Fermi National Accelerator Laboratory, Batavia, U.S.A.}

S. Abdullin, M. Albrow, M. Alyari, G. Apollinari, A. Apresyan, A. Apyan, S. Banerjee, L.A.T. Bauerdick, A. Beretvas, J. Berryhill, P.C. Bhat, G. Bolla ${ }^{\dagger}$, K. Burkett, J.N. Butler, A. Canepa, G.B. Cerati, H.W.K. Cheung, F. Chlebana, M. Cremonesi, J. Duarte, V.D. Elvira, J. Freeman, Z. Gecse, E. Gottschalk, L. Gray, D. Green, S. Grünendahl, O. Gutsche, R.M. Harris, S. Hasegawa, J. Hirschauer, Z. Hu, B. Jayatilaka, S. Jindariani, M. Johnson, U. Joshi, B. Klima, B. Kreis, S. Lammel, D. Lincoln, R. Lipton, M. Liu, T. Liu, R. Lopes De Sá, J. Lykken, K. Maeshima, N. Magini, J.M. Marraffino, S. Maruyama, D. Mason, P. McBride, P. Merkel, S. Mrenna, S. Nahn, V. O’Dell, K. Pedro, O. Prokofyev, G. Rakness, L. Ristori, B. Schneider, E. Sexton-Kennedy, A. Soha, W.J. Spalding, L. Spiegel, S. Stoynev, J. Strait, N. Strobbe, L. Taylor, S. Tkaczyk, N.V. Tran, L. Uplegger, E.W. Vaandering, C. Vernieri, M. Verzocchi, R. Vidal, M. Wang, H.A. Weber, A. Whitbeck University of Florida, Gainesville, U.S.A.

D. Acosta, P. Avery, P. Bortignon, D. Bourilkov, A. Brinkerhoff, A. Carnes, M. Carver, D. Curry, R.D. Field, I.K. Furic, J. Konigsberg, A. Korytov, K. Kotov, P. Ma, K. Matchev, H. Mei, G. Mitselmakher, D. Rank, D. Sperka, N. Terentyev, L. Thomas, J. Wang, S. Wang, J. Yelton

Florida International University, Miami, U.S.A.

Y.R. Joshi, S. Linn, P. Markowitz, J.L. Rodriguez

Florida State University, Tallahassee, U.S.A.

A. Ackert, T. Adams, A. Askew, S. Hagopian, V. Hagopian, K.F. Johnson, T. Kolberg, G. Martinez, T. Perry, H. Prosper, A. Saha, A. Santra, V. Sharma, R. Yohay 
Florida Institute of Technology, Melbourne, U.S.A.

M.M. Baarmand, V. Bhopatkar, S. Colafranceschi, M. Hohlmann, D. Noonan, T. Roy, F. Yumiceva

University of Illinois at Chicago (UIC), Chicago, U.S.A.

M.R. Adams, L. Apanasevich, D. Berry, R.R. Betts, R. Cavanaugh, X. Chen, O. Evdokimov, C.E. Gerber, D.A. Hangal, D.J. Hofman, K. Jung, J. Kamin, I.D. Sandoval Gonzalez, M.B. Tonjes, H. Trauger, N. Varelas, H. Wang, Z. Wu, J. Zhang

The University of Iowa, Iowa City, U.S.A.

B. Bilki ${ }^{61}$, W. Clarida, K. Dilsiz ${ }^{62}$, S. Durgut, R.P. Gandrajula, M. Haytmyradov, V. Khristenko, J.-P. Merlo, H. Mermerkaya ${ }^{63}$, A. Mestvirishvili, A. Moeller, J. Nachtman, H. Ogul ${ }^{64}$, Y. Onel, F. Ozok ${ }^{65}$, A. Penzo, C. Snyder, E. Tiras, J. Wetzel, K. Yi

Johns Hopkins University, Baltimore, U.S.A.

B. Blumenfeld, A. Cocoros, N. Eminizer, D. Fehling, L. Feng, A.V. Gritsan, P. Maksimovic, J. Roskes, U. Sarica, M. Swartz, M. Xiao, C. You

The University of Kansas, Lawrence, U.S.A.

A. Al-bataineh, P. Baringer, A. Bean, S. Boren, J. Bowen, J. Castle, S. Khalil, A. Kropivnitskaya, D. Majumder, W. Mcbrayer, M. Murray, C. Royon, S. Sanders, E. Schmitz, J.D. Tapia Takaki, Q. Wang

Kansas State University, Manhattan, U.S.A.

A. Ivanov, K. Kaadze, Y. Maravin, A. Mohammadi, L.K. Saini, N. Skhirtladze, S. Toda

Lawrence Livermore National Laboratory, Livermore, U.S.A.

F. Rebassoo, D. Wright

University of Maryland, College Park, U.S.A.

C. Anelli, A. Baden, O. Baron, A. Belloni, B. Calvert, S.C. Eno, C. Ferraioli, N.J. Hadley, S. Jabeen, G.Y. Jeng, R.G. Kellogg, J. Kunkle, A.C. Mignerey, F. Ricci-Tam, Y.H. Shin, A. Skuja, S.C. Tonwar

\section{Massachusetts Institute of Technology, Cambridge, U.S.A.}

D. Abercrombie, B. Allen, V. Azzolini, R. Barbieri, A. Baty, R. Bi, S. Brandt, W. Busza, I.A. Cali, M. D'Alfonso, Z. Demiragli, G. Gomez Ceballos, M. Goncharov, D. Hsu, Y. Iiyama, G.M. Innocenti, M. Klute, D. Kovalskyi, Y.S. Lai, Y.-J. Lee, A. Levin, P.D. Luckey, B. Maier, A.C. Marini, C. Mcginn, C. Mironov, S. Narayanan, X. Niu, C. Paus, C. Roland, G. Roland, J. Salfeld-Nebgen, G.S.F. Stephans, K. Tatar, D. Velicanu, J. Wang, T.W. Wang, B. Wyslouch

University of Minnesota, Minneapolis, U.S.A.

A.C. Benvenuti, R.M. Chatterjee, A. Evans, P. Hansen, S. Kalafut, Y. Kubota, Z. Lesko, J. Mans, S. Nourbakhsh, N. Ruckstuhl, R. Rusack, J. Turkewitz

University of Mississippi, Oxford, U.S.A.

J.G. Acosta, S. Oliveros 
University of Nebraska-Lincoln, Lincoln, U.S.A.

E. Avdeeva, K. Bloom, D.R. Claes, C. Fangmeier, R. Gonzalez Suarez, R. Kamalieddin, I. Kravchenko, J. Monroy, J.E. Siado, G.R. Snow, B. Stieger

State University of New York at Buffalo, Buffalo, U.S.A.

J. Dolen, A. Godshalk, C. Harrington, I. Iashvili, D. Nguyen, A. Parker, S. Rappoccio, B. Roozbahani

Northeastern University, Boston, U.S.A.

G. Alverson, E. Barberis, A. Hortiangtham, A. Massironi, D.M. Morse, D. Nash, T. Orimoto, R. Teixeira De Lima, D. Trocino, D. Wood

Northwestern University, Evanston, U.S.A.

S. Bhattacharya, O. Charaf, K.A. Hahn, N. Mucia, N. Odell, B. Pollack, M.H. Schmitt, K. Sung, M. Trovato, M. Velasco

University of Notre Dame, Notre Dame, U.S.A.

N. Dev, M. Hildreth, K. Hurtado Anampa, C. Jessop, D.J. Karmgard, N. Kellams, K. Lannon, N. Loukas, N. Marinelli, F. Meng, C. Mueller, Y. Musienko ${ }^{33}$, M. Planer, A. Reinsvold, R. Ruchti, G. Smith, S. Taroni, M. Wayne, M. Wolf, A. Woodard

The Ohio State University, Columbus, U.S.A.

J. Alimena, L. Antonelli, B. Bylsma, L.S. Durkin, S. Flowers, B. Francis, A. Hart, C. Hill, W. Ji, B. Liu, W. Luo, D. Puigh, B.L. Winer, H.W. Wulsin

Princeton University, Princeton, U.S.A.

S. Cooperstein, O. Driga, P. Elmer, J. Hardenbrook, P. Hebda, S. Higginbotham, D. Lange, J. Luo, D. Marlow, K. Mei, I. Ojalvo, J. Olsen, C. Palmer, P. Piroué, D. Stickland, C. Tully

University of Puerto Rico, Mayaguez, U.S.A.

S. Malik, S. Norberg

Purdue University, West Lafayette, U.S.A.

A. Barker, V.E. Barnes, S. Das, S. Folgueras, L. Gutay, M.K. Jha, M. Jones, A.W. Jung, A. Khatiwada, D.H. Miller, N. Neumeister, C.C. Peng, J.F. Schulte, J. Sun, F. Wang, W. Xie

Purdue University Northwest, Hammond, U.S.A.

T. Cheng, N. Parashar, J. Stupak

Rice University, Houston, U.S.A.

A. Adair, B. Akgun, Z. Chen, K.M. Ecklund, F.J.M. Geurts, M. Guilbaud, W. Li, B. Michlin, M. Northup, B.P. Padley, J. Roberts, J. Rorie, Z. Tu, J. Zabel

University of Rochester, Rochester, U.S.A.

A. Bodek, P. de Barbaro, R. Demina, Y.t. Duh, T. Ferbel, M. Galanti, A. Garcia-Bellido, J. Han, O. Hindrichs, A. Khukhunaishvili, K.H. Lo, P. Tan, M. Verzetti

The Rockefeller University, New York, U.S.A.

R. Ciesielski, K. Goulianos, C. Mesropian 
Rutgers, The State University of New Jersey, Piscataway, U.S.A.

A. Agapitos, J.P. Chou, Y. Gershtein, T.A. Gómez Espinosa, E. Halkiadakis, M. Heindl,

E. Hughes, S. Kaplan, R. Kunnawalkam Elayavalli, S. Kyriacou, A. Lath, R. Montalvo,

K. Nash, M. Osherson, H. Saka, S. Salur, S. Schnetzer, D. Sheffield, S. Somalwar, R. Stone,

S. Thomas, P. Thomassen, M. Walker

University of Tennessee, Knoxville, U.S.A.

A.G. Delannoy, M. Foerster, J. Heideman, G. Riley, K. Rose, S. Spanier, K. Thapa

Texas A\&M University, College Station, U.S.A.

O. Bouhali ${ }^{66}$, A. Castaneda Hernandez ${ }^{66}$, A. Celik, M. Dalchenko, M. De Mattia, A. Delgado, S. Dildick, R. Eusebi, J. Gilmore, T. Huang, T. Kamon ${ }^{67}$, R. Mueller, Y. Pakhotin, R. Patel, A. Perloff, L. Perniè, D. Rathjens, A. Safonov, A. Tatarinov, K.A. Ulmer

Texas Tech University, Lubbock, U.S.A.

N. Akchurin, J. Damgov, F. De Guio, P.R. Dudero, J. Faulkner, E. Gurpinar, S. Kunori, K. Lamichhane, S.W. Lee, T. Libeiro, T. Peltola, S. Undleeb, I. Volobouev, Z. Wang

Vanderbilt University, Nashville, U.S.A.

S. Greene, A. Gurrola, R. Janjam, W. Johns, C. Maguire, A. Melo, H. Ni, K. Padeken, P. Sheldon, S. Tuo, J. Velkovska, Q. Xu

\section{University of Virginia, Charlottesville, U.S.A.}

M.W. Arenton, P. Barria, B. Cox, R. Hirosky, M. Joyce, A. Ledovskoy, H. Li, C. Neu, T. Sinthuprasith, Y. Wang, E. Wolfe, F. Xia

\section{Wayne State University, Detroit, U.S.A.}

R. Harr, P.E. Karchin, J. Sturdy, S. Zaleski

\section{University of Wisconsin - Madison, Madison, WI, U.S.A.}

M. Brodski, J. Buchanan, C. Caillol, S. Dasu, L. Dodd, S. Duric, B. Gomber, M. Grothe, M. Herndon, A. Hervé, U. Hussain, P. Klabbers, A. Lanaro, A. Levine, K. Long, R. Loveless, G.A. Pierro, G. Polese, T. Ruggles, A. Savin, N. Smith, W.H. Smith, D. Taylor, N. Woods

\section{†: Deceased}

1: Also at Vienna University of Technology, Vienna, Austria

2: Also at State Key Laboratory of Nuclear Physics and Technology, Peking University, Beijing, China

3: Also at Universidade Estadual de Campinas, Campinas, Brazil

4: Also at Universidade Federal de Pelotas, Pelotas, Brazil

5: Also at Université Libre de Bruxelles, Bruxelles, Belgium

6: Also at Institute for Theoretical and Experimental Physics, Moscow, Russia

7: Also at Joint Institute for Nuclear Research, Dubna, Russia

8: Now at Cairo University, Cairo, Egypt

9: Also at Zewail City of Science and Technology, Zewail, Egypt

10: Also at Université de Haute Alsace, Mulhouse, France

11: Also at Skobeltsyn Institute of Nuclear Physics, Lomonosov Moscow State University, Moscow, Russia 
12: Also at CERN, European Organization for Nuclear Research, Geneva, Switzerland

13: Also at RWTH Aachen University, III. Physikalisches Institut A, Aachen, Germany

14: Also at University of Hamburg, Hamburg, Germany

15: Also at Brandenburg University of Technology, Cottbus, Germany

16: Also at MTA-ELTE Lendület CMS Particle and Nuclear Physics Group, Eötvös Loránd University, Budapest, Hungary

17: Also at Institute of Nuclear Research ATOMKI, Debrecen, Hungary

18: Also at Institute of Physics, University of Debrecen, Debrecen, Hungary

19: Also at Indian Institute of Technology Bhubaneswar, Bhubaneswar, India

20: Also at Institute of Physics, Bhubaneswar, India

21: Also at University of Visva-Bharati, Santiniketan, India

22: Also at University of Ruhuna, Matara, Sri Lanka

23: Also at Isfahan University of Technology, Isfahan, Iran

24: Also at Yazd University, Yazd, Iran

25: Also at Plasma Physics Research Center, Science and Research Branch, Islamic Azad University, Tehran, Iran

26: Also at Università degli Studi di Siena, Siena, Italy

27: Also at INFN Sezione di Milano-Bicocca; Università di Milano-Bicocca, Milano, Italy

28: Also at Purdue University, West Lafayette, U.S.A.

29: Also at International Islamic University of Malaysia, Kuala Lumpur, Malaysia

30: Also at Malaysian Nuclear Agency, MOSTI, Kajang, Malaysia

31: Also at Consejo Nacional de Ciencia y Tecnología, Mexico city, Mexico

32: Also at Warsaw University of Technology, Institute of Electronic Systems, Warsaw, Poland

33: Also at Institute for Nuclear Research, Moscow, Russia

34: Now at National Research Nuclear University 'Moscow Engineering Physics Institute' (MEPhI), Moscow, Russia

35: Also at St. Petersburg State Polytechnical University, St. Petersburg, Russia

36: Also at University of Florida, Gainesville, U.S.A.

37: Also at P.N. Lebedev Physical Institute, Moscow, Russia

38: Also at California Institute of Technology, Pasadena, U.S.A.

39: Also at Budker Institute of Nuclear Physics, Novosibirsk, Russia

40: Also at Faculty of Physics, University of Belgrade, Belgrade, Serbia

41: Also at University of Belgrade, Faculty of Physics and Vinca Institute of Nuclear Sciences, Belgrade, Serbia

42: Also at Scuola Normale e Sezione dell'INFN, Pisa, Italy

43: Also at National and Kapodistrian University of Athens, Athens, Greece

44: Also at Riga Technical University, Riga, Latvia

45: Also at Universität Zürich, Zurich, Switzerland

46: Also at Stefan Meyer Institute for Subatomic Physics (SMI), Vienna, Austria

47: Also at Adiyaman University, Adiyaman, Turkey

48: Also at Istanbul Aydin University, Istanbul, Turkey

49: Also at Mersin University, Mersin, Turkey

50: Also at Cag University, Mersin, Turkey

51: Also at Piri Reis University, Istanbul, Turkey

52: Also at Izmir Institute of Technology, Izmir, Turkey

53: Also at Necmettin Erbakan University, Konya, Turkey

54: Also at Marmara University, Istanbul, Turkey

55: Also at Kafkas University, Kars, Turkey 
56: Also at Istanbul Bilgi University, Istanbul, Turkey

57: Also at Rutherford Appleton Laboratory, Didcot, United Kingdom

58: Also at School of Physics and Astronomy, University of Southampton, Southampton, United Kingdom

59: Also at Instituto de Astrofísica de Canarias, La Laguna, Spain

60: Also at Utah Valley University, Orem, U.S.A.

61: Also at Beykent University, Istanbul, Turkey

62: Also at Bingol University, Bingol, Turkey

63: Also at Erzincan University, Erzincan, Turkey

64: Also at Sinop University, Sinop, Turkey

65: Also at Mimar Sinan University, Istanbul, Istanbul, Turkey

66: Also at Texas A\&M University at Qatar, Doha, Qatar

67: Also at Kyungpook National University, Daegu, Korea 